核嘱合研究第 1.0 巻第 2 号 1963 年 2 月

Moment Method for Wave

Phenomena in a Plasma

Effect of Two - Body Collisions
on the Plasma Oscillation

小笠原正忠 (東大理)

( 1963 年 1 月 16 日受理)

目

次

$\$$ I Introduction

$\$ 2$ Preliminary

$\$ 3$ Collision Term

$\$ 4$ Dispersion Relation

$\$ 5$ Discussion of the Dispersion Relation

\$ 6 Integrated iloment Equation

$\$ 7$ Moment Wave

$\$ 8$ Effect of Ion-Electron Collision

Appendix A

". B Derivation of a Recurrence Formula

" C Evaluation of $\left(\varphi_{\mathbf{p} \ell}, \mathrm{J}_{e-i} \varphi_{q e}\right)$

Reference

* M. Ogasawara,

Department of Physics,

University of Tokyo. 
$\$ 1$. Introduction

Plasma のkinetic theory の問題を大別すると，

(1) 熱平衡状態

2) 定常状態の輸送現象

(3) 空間的飞一様な速度分布の緩和

(4) 平衡状態の方りりの小変動の伝播

の上5に索る。(3灶初期速度分布 $f(\overrightarrow{\mathrm{v}}, t=0)$ が与えられたとを

$$
\frac{\partial f}{\partial t}=\left(\frac{\partial f}{\partial t}\right) \operatorname{col} 1
$$

を調へるてとが問題である。 $f=f^{(0)}(1+\phi)\left(f^{(0)}=\right.$ 平衡分布 $)$ とおいてゆとついて線形化 ナると

$$
\frac{\partial \varphi}{\partial t}=-n_{0} \mathrm{~J} \phi
$$

となる。的は線形対称な collision operatorである。このようにして relaxation の問題恃 operator Jの固有值問題に帰着される。乙れは MaxwelI molecule に対してのみ解けている。 coulomb force力場合は I ( reference 1 ) をI と書く）に於いて, 正確な固有值を求めるてとの困難さが示され, 又一部の近似固有值が求めら れている。

(4)は

$$
\left(\frac{\partial}{\partial t}+\overrightarrow{\mathbf{v}} \cdot \vec{\nabla}_{x}-\frac{\mathbf{e}}{m} \overrightarrow{\mathrm{E}} \cdot \overrightarrow{\boldsymbol{\nabla}} \overrightarrow{\mathbf{a}}\right)^{\phi}=-n_{o} \mathrm{~J} \phi
$$

によって支配される。たとえMaxwell molecule の場合です4灶完全には决をらない。 Mott-Smith, Wang Chang and Uhlenbeck

2) 仕 $\phi=\sum a_{i} \Psi_{i}(\overrightarrow{\mathrm{v}})\left[\Psi_{i} \overrightarrow{(\mathrm{v})}\right.$ は Maxwel工 molecule の場台の固有函数 $\left.{ }^{\prime}\right)$ Jの形の解を見出そうとしたが(1、3)の 左欢第 2 項のために, $\dot{a}_{i}$ 方程式に $a_{j}(j>i)$ か人って来て, いわゅる階級方程式になる。 従って, collision operator の固有值問題が解けたとしても, wave の問題を完全に 解くことは出来ない。

collisionlessの場合でを上様にすると階級方程式になって了う。しかし（1、3）を Fourier 変換して形式的に $\emptyset$ を求めてから速度積分して moment を計算すると, 前の様な 
小笠原

左辺第 2 項による higher modeの介入をさけることが出来て, exactな dispersion relation が得られる。〔\$4]

しかし collision を考慮すると同様の手続を採ってもJが operator支ので（1、3）の 左辺第 2 項と同じ事情に去り exactな dispersion relation は得られない。样 な複雑さのために, plasma 振動に対して collision の効果を正しくとり入れている理論 はない。ててで正しく”と云う意味は Boltzmann collision term の範团内での話で ある。Boltzmann方程式には相関効果沬含まれていないしかし coulomb力の様な longrange force の場合は相関効果を無視し得ないであろう。此の様な意図のもとに最近 plasmak対するkinetic eqの研究が盛んになって来た。 ${ }^{3}$ ) Y.H.Ichikawa ${ }^{4}$ ), C.R.Willis ${ }^{5}$ ) 等恃 2 体相関函数支考慮した kinetic eq. ‘ set up LCplasma oscillationの研究を行なっている。〔\$ 5 〕

此の新しい研究との比較の意味でも2 体 collision pracess の勃果を exactに与える ことは意義深らととと思われる。本論文では Boltzmann-VIasov eq. から出発し， collision frequency が plasma frequency K比して小さい場合を扱い, 長波長 極限,すなわち波数居の 2 乗の order 栗で exact結果を与える。

\section{§2. Preliminary}

(8) Definition of moment

平衡からのはずれが小さい時, 電子の速度分布函数 $f(\overrightarrow{\mathrm{v}})$ を

$$
f=f^{(0)}+f^{(1)}, \quad f^{(1)}=f^{(0)} \phi, \quad|\phi| \ll 1
$$

の様に展開する。 $\overrightarrow{\mathrm{v}}$ は電子の速度, $f^{(0)}$ 壮平衡状態の分布で MaXwel工分布 :

$$
f^{(0)}(\overrightarrow{\mathbf{v}})=n_{0}\left(\frac{m}{2 \pi \kappa \mathrm{T}}\right)^{\frac{3}{2}} \mathbf{e} \times \mathbf{p}\left(-\frac{m v^{2}}{2 \kappa \mathrm{T}_{0}}\right)
$$

である。 $m, n_{o}, \mathrm{~T}_{o}$ ，火はそれぞれ電子の mass, number density, temperature, Boltzmann constant を意味する。

次の様な直交函数系導入する。门 )

$$
\Psi_{\mathrm{r} e}(\overrightarrow{\mathrm{v}})=\frac{\varphi_{\mathbf{r}_{e}}}{\mathbf{N}_{\mathbf{r} e}}=\frac{1}{\mathrm{~N}_{\mathrm{r} e}} \xi^{e} \mathrm{P}_{e}\left(\frac{\xi_{\mathrm{z}}}{\xi}\right) \mathrm{S}_{e+\frac{1}{2}}^{(\mathbf{r})}\left(\xi^{2}\right) \quad(2.3)
$$




$$
\begin{gathered}
\dot{\xi}^{2}=\frac{m}{2 \kappa \mathrm{T}_{o}} v^{2} \equiv(\alpha v)^{2}, \\
\mathrm{~N}_{\mathrm{re}}^{2}=\frac{2}{2 \ell+1} \frac{1}{\sqrt{\pi} \mathrm{r} !} \Gamma\left(\ell+\mathrm{r}+\frac{3}{2}\right)
\end{gathered}
$$

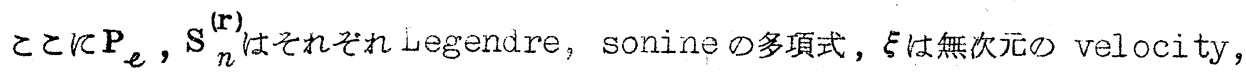
$\mathbf{N}_{\mathbf{r}} e^{\text {は }}$ normalization constant 意味する。velocity $\overrightarrow{\mathbf{v}}$ の任意函数 $\phi(\overrightarrow{\mathbf{v}})$, $\psi(\vec{v})$ ○内積を

$$
(\phi, \psi)=\frac{1}{n_{0}} \int f^{(0)} \phi \psi d \overrightarrow{\mathrm{v}}
$$

で定義する時，函数系 $\left\{\Psi_{\mathrm{r} e}\right\}$ の直交関係

$$
\left(\Psi_{r e}, \Psi_{r^{\prime} e^{\prime}}\right)=\delta r_{r^{\prime}} \delta_{e} e^{\prime}
$$

が成り立つ。 $\left\{\Psi_{\mathrm{r} e}\right\}$ tbase にとり分布の平衡らのはずれ $\phi(\overrightarrow{\mathrm{v}}, \overrightarrow{\mathrm{x}}, t)$ を

$$
\begin{aligned}
& \varphi(\overrightarrow{\mathrm{v}}, \overrightarrow{\mathrm{x}}, t)=\sum a_{\mathrm{re}}(\overrightarrow{\mathrm{x}}, t) \Psi_{\mathrm{re}}(\overrightarrow{\mathrm{v}}) \\
& a_{\mathrm{re}}(\overrightarrow{\mathrm{x}}, t)=\left(\Psi_{\mathrm{re}}, \phi\right)=\frac{1}{n_{0}} \int f^{(1)} \Psi_{\mathrm{re}} d \overrightarrow{\mathrm{v}}
\end{aligned}
$$

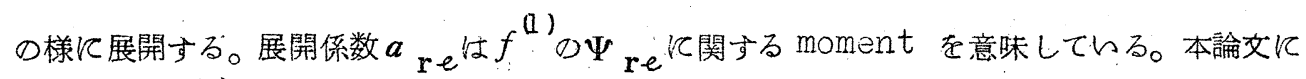
於いては, $f^{(1)}$ の代りに moment扱ら。

(b) Moment \& Macroscopic Quantity

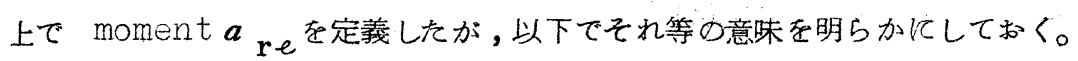

平衡状態に於ける量に suff'ix又は superscript 0 を, 平衡からのはずれを意味する量 K primeを附し,それを無次元化したものに ripple mark〜を附す。

$$
\begin{aligned}
& \text { Number density } \quad n=n_{0}+n^{\prime}=n_{0}(1+\widetilde{n}) \\
& \text { Wacroscopic velocity } \quad \vec{u}=0+\vec{u}^{\prime}=\alpha^{-1} \tilde{u} \\
& \text { Temperature } \\
& \mathrm{T}=\mathrm{T}_{o}+\mathrm{T}^{\prime}=\mathrm{T}_{o}(1+\widetilde{\mathrm{T}})(2,10) \\
& \text { Pressure tensor } \\
& \mathrm{P}_{i j}=\mathrm{P}_{i j}^{o}+\mathrm{P}_{i j}^{\prime}=n_{i b^{k}} \mathrm{~T}_{o}\left(1+\widetilde{\mathrm{p}}_{i j}\right) \\
& \text { Heat flow vector } \\
& \vec{q}=0+\vec{q}^{\prime}=\frac{1}{2} n_{c} m \alpha^{-3} \underset{\vec{q}}{\widetilde{q}}
\end{aligned}
$$


これらの量を分布 $f$ を用いて計算し, moment $a$ reとの関係を見る。

[number density]

$$
n=\int f \overrightarrow{d \vec{v}}=\int f^{(0)} d \overrightarrow{\mathrm{v}}+\int f^{(1)} d \overrightarrow{\mathrm{v}}=n_{o}+n_{o}(\varphi, 1) .
$$

従って

$$
\widetilde{n}=(\Phi, 1)=a_{00}
$$

[macroscopic velocity]

$$
\begin{aligned}
& u_{z}^{\prime}=\frac{1}{n_{0}} \int f^{(0)} \phi v_{z} d \overrightarrow{\mathrm{v}}=\frac{\alpha^{-1} \mathrm{~N}_{O 1}}{n_{0}}\left(\phi, \Psi_{0,}\right) \\
& \therefore \widetilde{u}_{z}=\mathbb{N}_{O_{1}}\left(\phi, \Psi_{a_{1}}\right)=\frac{1}{\sqrt{2}} a_{o 1}
\end{aligned}
$$

[kinetic temperature ]

$$
\begin{aligned}
& 3 n \kappa \mathrm{T}=m \int f v^{2} d \overrightarrow{\mathrm{v}}, \\
& 3 n \kappa \mathrm{T}=3 n_{o} \kappa \mathrm{T}_{o}(1+\widetilde{n}+\widetilde{\mathrm{T}}) .
\end{aligned}
$$

従って. $3 n_{o} \kappa \mathrm{T}_{0} \widetilde{\mathrm{T}}=m \int f^{(0)} \phi v^{2} d \overrightarrow{\mathrm{v}}-3 n_{0} \kappa \mathrm{T}_{0} \tilde{n}$

$$
\begin{gathered}
=-2 n_{0} \kappa \mathrm{T}_{0} \mathrm{~N}_{10}\left(\phi, \Psi_{10}\right) . \\
\therefore \widetilde{\mathrm{T}}=-\frac{2}{3} \mathrm{~N}_{10}\left(\phi, \Psi_{10}\right)=-\sqrt{\frac{2}{3} a_{10}}
\end{gathered}
$$

[Pressure tensor ]は macroscopic velocity に乗った座標系で見た momentum transportとして定義される。

$$
p_{i j}=m \int f(\overrightarrow{\mathrm{v}}-\overrightarrow{\mathrm{u}})_{i}(\vec{v}-\overrightarrow{\mathrm{u}})_{j} d \overrightarrow{\mathrm{v}}
$$

Iinearizationると

$$
\begin{aligned}
& p_{z z}=p_{z z}^{0}+p_{z z}^{\prime} \\
& p_{z z}^{\prime}=m \int f^{(0)} \phi v_{z}^{2} d \vec{v}
\end{aligned}
$$

今 scalar pressure $\mathbf{p}^{\prime}$ \& stress tensor $\mathbf{S}_{i}^{\prime}$,

$$
p^{\prime}=\frac{1}{3} \mathrm{~T}_{\mathbf{r}} p_{i j}^{\prime}=\frac{1}{3} m \int f^{(0)} \phi v^{2} d \vec{v}
$$


Moment Method for Wave Phenomena in a Plasma

$$
\mathrm{s}_{i j}^{\prime}=p_{i j}^{\prime}-p^{\prime} \delta_{i j}
$$

によって定義すると

$$
\begin{aligned}
\mathrm{S}_{z z}^{\prime} & =p_{z z}^{\prime}-p=m \int f^{(0)} \phi\left(v_{z}^{2}-\frac{v^{2}}{3}\right) d \overrightarrow{\mathrm{v}} \\
& =\frac{4}{3} n_{0} \kappa \mathrm{T}_{0} \mathrm{~N}_{O_{2}}\left(\phi, \Psi_{o_{2}}\right) \\
\therefore & \widetilde{\mathrm{S}}_{z z}=\frac{2}{\sqrt{3}}\left(\phi, \Psi_{O_{2}}\right)=\frac{2}{\sqrt{3}} a_{o_{2}}
\end{aligned}
$$

[ heat flow vector]花で動いている座標采で定義される。

$$
q_{z}=\frac{1}{2} m \int f(\overrightarrow{\mathrm{v}}-\vec{u})^{2}(\overrightarrow{\mathrm{v}}-\vec{u})_{z} d \overrightarrow{\mathrm{v}}
$$

Iinearization して

$$
\begin{aligned}
g_{z}^{\prime} & =-\frac{5}{2} n_{o} \kappa T_{o} u_{z}+\frac{1}{2} m \int f^{(0)} \phi v^{2} v_{z} d \overrightarrow{\mathrm{v}} \\
& =-\frac{1}{2} n_{0} m \alpha^{-3} \mathrm{~N}_{11}\left(\phi, \Psi_{11}\right) \\
\therefore \widetilde{q}_{z} & =-\frac{\sqrt{5}}{2}\left(\phi, \Psi_{11}\right)=-\frac{\sqrt{5}}{2} a_{11}
\end{aligned}
$$

以上ををとると下表のよらになる。

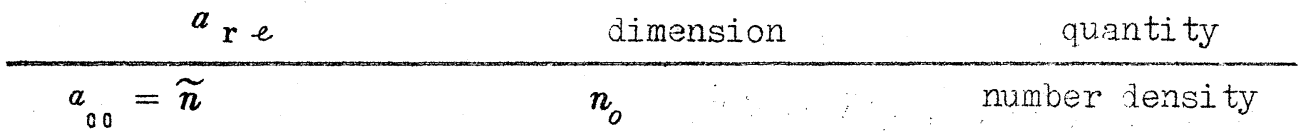

$$
\begin{aligned}
& a_{01}=\sqrt{2} \widetilde{u}_{z} \\
& \left(\frac{2 \kappa \mathrm{T}_{0}}{m}\right)^{1 / 2} \quad \text { macroscopic } \\
& a_{10}=-\sqrt{\frac{3}{2}} \widetilde{T} \quad \mathrm{~T}_{0} \quad \text { termperature } \\
& a_{02}=\frac{\sqrt{3}}{2} \widetilde{S}_{z z} \quad n_{0} \kappa \mathrm{T}_{0} \quad \text { stress tensor } \\
& a_{11}=-\frac{2}{\sqrt{5}} \widetilde{q}_{z} \quad \frac{1}{2} n_{0} m\left(\frac{2 \kappa \mathrm{T}_{0}}{m} ; \quad\right. \text { heat flow }
\end{aligned}
$$


\$3. Collision Term

Plasma に於ける輸送現象の扱いの場合, collision term . 快

Boltzman's type

Fokker - Planck type

のレつれかが正確な形で用いられている。しかし，plasma oscillationの理論では

collision term を正確な形で用いたるのはない。多体相関効果のplasma oscillation への奇与が問題になって来た昨今，2体衝乫の寄与を正確に与えるてとは非常に望をれるとてろ である。

以下簡単に今迄用らられて来た collision term をみるととにする。最も多く用いられ るのは

$$
\frac{\partial \phi}{\partial t}=-\nu \phi
$$

である。 $\left.{ }^{6}\right)$ 乙れは relaxation feature を含んでいるが, single relaxation constant theoryでありIで示したょうに余り良い近似でない。文保存則を满していないの で,電子一電子衝突を扱うことは出来ない。

Bhatnager, Gross and $\mathrm{Krook}^{7}$ ) は保存則をみたすような model collision term set upして分散式を与えている。

$$
\frac{\partial \phi}{\partial t}=\frac{\eta_{0}}{\sigma}\left[-\phi+\nu+\frac{m}{\kappa \mathrm{T}_{0}} \overrightarrow{\mathrm{v}} \cdot \overrightarrow{\mathrm{q}}+\tau\left(\frac{m v^{2}}{2 \kappa \mathrm{T}_{0}}-\frac{3}{2}\right)\right],
$$

ここに

$$
\begin{aligned}
& \nu=\int \phi \mathrm{F} d \overrightarrow{\mathrm{v}}, \overrightarrow{\mathrm{q}}=\int \overrightarrow{\mathrm{v}} \phi \mathrm{F} d \overrightarrow{\mathrm{v}}, \\
& \nu+\tau=\frac{m}{3 \kappa \mathrm{T}_{0}} \int v^{2} \phi \mathrm{F} d \overrightarrow{\mathrm{v}}, \\
& \mathrm{F}=\left(\frac{m}{2 \pi \kappa \mathrm{T}}\right)^{\frac{3}{2}} \exp \left(-\frac{m v^{2}}{2 \kappa \mathrm{T}}\right)
\end{aligned}
$$

$\frac{n_{o}}{\sigma}$ か collision frequency になっている。てのmodel ま single relaxation constant theoryであるが, このために数学的に簡単化されて, collision frequency のどんな值に対してる成り立つ分散式が得られる。 
Moment Method for Wave Phenomena in a Plasma

一般分布のmodeによって relaxation constant 仕異なる。') 㵊近 Johnston 壮分布 $f$.

$$
\begin{aligned}
& f=\sum_{\ell m s} f_{\ell m s}\left(v^{2}, \mathbf{r}, t\right) \mathrm{Y}_{\ell m s}(\theta, \varphi) \\
& =f_{o}+\frac{\mathbf{f}_{1} \cdot \vec{v}}{v}+\frac{\mathbf{f}_{2}: \vec{v} \vec{v}}{v^{2}}+\cdots \cdots, \quad(3,3), \\
& \mathrm{Y}_{\ell m s}(\theta, \varphi)=\mathrm{P}_{\ell}^{m}(\cos \theta)\left(\delta_{o s} \cos m \varphi+\delta_{1 s} \sin m \varphi\right), \\
& \mathbf{f} \ell: \ell \text { 階の tensor }
\end{aligned}
$$

と展開し $\dot{f}_{0}, \dot{f}, \dot{f}_{2}$ 亿対する3つの方程式を基礎方程式として electric conductivity tensor を求好ている。それぞれの衝乫項 $\overrightarrow{\mathrm{c}}_{\boldsymbol{\ell}}$

$$
\mathrm{C}_{e}=-\nu_{e} \mathbf{f}_{e}
$$

とかいているが, 最後の expression は, $\nu_{0}=\nu_{1}=\nu_{2}$ として出している。分布 $\mathbf{f}$ の展

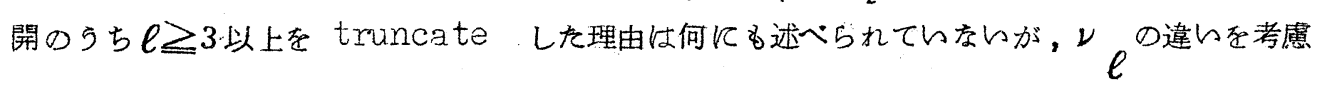

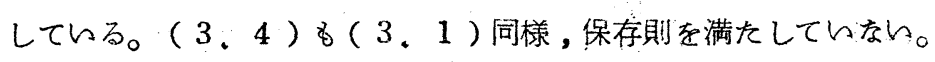

一方 coulomb force に特徽的な small angle scatteringt考慮する意味で， Lenard, and Bernstein $\left.{ }^{9}\right)$ 又 Logunov ${ }^{10}$ ) 恃速度空間k於ける Fokker-Planck eq.

$$
\begin{aligned}
& \frac{\partial f^{(1)}}{\partial t}=\beta \frac{\partial}{\partial \vec{v}} \cdot\left[\vec{v} f^{(1)}+v_{0}^{2} \frac{\partial f(1)}{\partial \vec{v}}\right], \\
& f=f^{(0)}+f^{(1)}, \\
& \beta: \quad \text { effective collision frequency, } \\
& v_{0}: \quad \text { root mean square speed }
\end{aligned}
$$

を用いているが結果杜 single relaxation constant theory と同じである。

以上種々の model collision term につんて述へたが, 我々は近似なしに Boltzmann collision term を用いる。 linearized collision term は')

$$
\frac{\partial \phi}{\partial t}=-n_{o} \mathrm{~J} \phi \text {. }
$$


小笠原

Jは collision operator で次の様に定義される。

$$
\begin{aligned}
& \mathrm{J} \phi= \frac{1}{n_{o}} \iint f^{(0)}\left(\overrightarrow{\mathrm{v}}_{2}\right)\left[\Phi\left(\overrightarrow{\mathrm{v}}_{1}\right)+\Phi\left(\overrightarrow{\mathrm{v}}_{2}\right)-\Phi\left(\overrightarrow{\mathrm{v}}_{1}^{\prime}\right)-\Phi\left(\overrightarrow{\mathrm{v}}_{2}^{\prime}\right)\right] \\
& \times g I(g, \chi) d \Omega d \vec{v}_{2} . \\
& \vec{v}_{i}^{\prime}: \text { 衝突後の velocity } \\
& g: \text { relative speed }\left|\vec{v}_{1}-\vec{v}_{2}\right|=\left|\vec{v}_{1}^{\prime}-\vec{v}_{2}^{\prime}\right| \\
& \chi \quad \text { : 散乱角, } \\
& \mathrm{I}(g, \chi) \text { : 散乱の断面積, } \\
& d \Omega \text { : 散乱の立体角, }
\end{aligned}
$$

展開 $(2,8)$ を $(3,6)$ 飞代入すると

$$
\begin{aligned}
&\left.\frac{\partial \phi}{\partial t}\right)_{\mathrm{coll}}=n_{0} \sum_{\mathrm{r} e} a_{\mathrm{r} e^{\mathrm{J}} \Psi_{\mathrm{r} e}} \\
&=-\sum_{\mathrm{r} s e} \lambda_{\mathrm{r} s}^{\varphi}{ }^{a}{ }_{\mathrm{re}} \Psi_{s \ell}, \\
& \lambda \underset{\mathrm{r} s}{\varphi)}=n_{0}\left(\Psi_{\mathrm{r} e}, \mathrm{~J} \Psi_{s e}\right)=\lambda \underset{s r}{\varphi} .
\end{aligned}
$$

$\lambda{ }_{\mathrm{r} s}^{(\ell)}$ は $(3 \cdot 7)$ を用いて計算出来る量である。

collision の種類 (電子一電子, 電子一イオン) 仗 crs section $I(g, \chi) k$ 含まれている。

従って ( 3.8$)$ は一般的な collision term をあらわす。

$\S 4$. Dispersion Relation

電子の縦振動を考える。イオン壮 charge neutrality を保つように一様にぬりつふさ れている。ての section ではイオン一電子衝笑は考えないととにする。イオンの効果は 8 で扱われる。基礎方程式は

$$
\frac{\partial f}{\partial t}+\overrightarrow{\mathbf{v}} \cdot \frac{\partial f}{\partial \overrightarrow{\mathbf{x}}}-\frac{\mathbf{e}}{m} \overrightarrow{\mathbf{E}} \cdot \frac{\partial f}{\partial \overrightarrow{\mathbf{v}}}=\left(\frac{\partial f}{\partial t}\right)_{\operatorname{col} 1}, \quad(4,1)
$$


Moment Method for Wave Phenomena in a Plasma

$$
d i v \overrightarrow{\mathrm{E}}=4 \pi e\left(n_{o}-\int f d \overrightarrow{\mathrm{v}}\right)
$$

ここに電子の chargeは一主採られている。

これらを( 2 ・1) ( 2 ・2)を用いて Iinearizationすると

$$
\begin{aligned}
& \frac{\partial \phi}{\partial t}+\overrightarrow{\mathrm{v}} \cdot \frac{\partial \phi}{\partial \overrightarrow{\mathrm{x}}}+2 \alpha^{2} \frac{e}{m} \overrightarrow{\mathrm{E}} \cdot \overrightarrow{\mathrm{v}}=-n_{o} \mathrm{~J} \phi, \\
& d i v \overrightarrow{\mathrm{E}}=-4 \pi \mathrm{e} \int \mathrm{f}^{(0)} \phi d \overrightarrow{\mathrm{v}} .
\end{aligned}
$$

ててで（3.6)で与えられお collision termを用的。との方程式系に対し

$$
\left(\begin{array}{c}
\phi \\
\overrightarrow{\mathbf{E}}
\end{array}\right)=\left(\begin{array}{c}
\phi \overrightarrow{\mathbf{k}} \\
\overrightarrow{\mathbf{E}} \overrightarrow{\mathbf{k}}
\end{array}\right) \exp (i \overrightarrow{\mathbf{k}} \cdot \overrightarrow{\mathbf{x}}-i \omega t)
$$

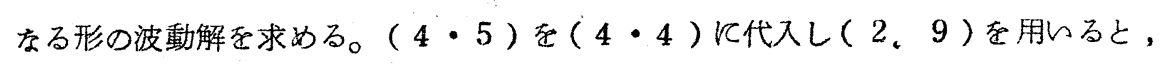

$$
\begin{aligned}
& \frac{e}{m} \overrightarrow{\mathrm{E}} \overrightarrow{\mathbf{k}}=\omega_{o}^{2} \frac{i \overrightarrow{\mathbf{k}}}{h^{2}} a_{00}^{\overrightarrow{\mathbf{k}}}, \\
& \omega_{o}^{2}=\frac{4 \pi n_{0} \mathrm{e}^{2}}{m} \cdots \cdots \text { plasma frequency. }
\end{aligned}
$$

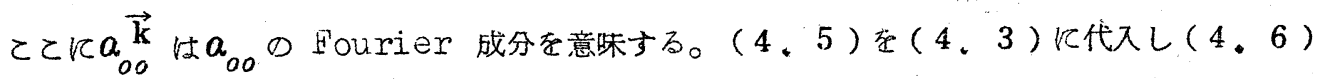

(3.8) 的ると，

$$
\begin{gathered}
i(\omega-\overrightarrow{\mathbf{k}} \cdot \overrightarrow{\mathbf{v}}) \phi_{\overrightarrow{\mathbf{k}}}=i \overrightarrow{\mathbf{k}} \cdot \overrightarrow{\mathbf{v}}\left(\frac{\ell_{D}}{h}\right)^{2} a_{o o}^{\overrightarrow{\mathbf{k}}}+\sum_{n s t} \lambda_{n s}^{(t)} \Psi_{s t} a_{n t}^{\overrightarrow{\mathbf{k}}}, \\
\therefore \phi_{\overrightarrow{\mathbf{k}}}=\frac{\overrightarrow{\mathbf{k}} \cdot \overrightarrow{\mathbf{v}}}{\omega-\overrightarrow{\mathbf{k}} \cdot \overrightarrow{\mathbf{v}}}\left(\frac{\ell_{D}}{\ell}\right)^{2} a_{o o}^{\overrightarrow{\mathbf{k}}}-i \sum_{n s t} \lambda_{n s}^{(H)} \frac{\Psi_{s t}}{\omega-\overrightarrow{\mathbf{k}} \cdot \overrightarrow{\mathbf{v}}} a_{n t}^{\overrightarrow{\mathbf{k}}}, \\
\ell_{\mathrm{D}}^{2}=\frac{4 \pi n_{o} \mathrm{e}^{2}}{s \mathrm{~T}_{o}}
\end{gathered}
$$

(4.7) の両辺に $\frac{1}{n_{0}} \int d \overrightarrow{\mathrm{v}} f^{\varphi)} \Psi_{\mathrm{r} e}$ を operateして (2.9)を考えると

$$
a_{\mathbf{r} e}^{\overrightarrow{\mathbf{k}}}=\alpha_{\mathbf{r} e} a_{o o}^{\overrightarrow{\mathbf{k}}}+\sum_{s t} \beta_{\mathrm{rest}} a_{s t}^{\overrightarrow{\mathbf{k}}}
$$


ことR

$$
\left.\begin{array}{l}
\alpha_{\mathrm{r} e}=\left(\frac{\ell_{\mathrm{D}}}{t^{2}}\right)^{2}\left(\frac{\overrightarrow{\mathbf{k}} \cdot \overrightarrow{\mathrm{v}}}{\omega-\overrightarrow{\mathbf{k}} \cdot \overrightarrow{\mathbf{v}}}, \Psi_{\mathrm{r} e}\right), \\
\beta_{\mathrm{rest}}=-i \sum_{n} \lambda_{n s}^{(t)}\left(\frac{\Psi_{\mathbf{r e}}}{\omega-\overrightarrow{\mathbf{k}} \cdot \overrightarrow{\mathrm{v}}}, \Psi_{n t}\right)
\end{array}\right\}
$$

$\overrightarrow{\mathrm{k}}$ 方向を $\mathrm{z}$ 軸に採り

$$
\lambda=i \frac{\alpha \omega}{k}
$$

とおくと(4、9)壮

$$
\left.\begin{array}{l}
\alpha_{\mathrm{r} e}=-\left(\frac{\omega_{0}}{\omega}\right)^{2} 2 \lambda^{2}\left(\frac{i \xi_{z}}{\lambda-i \xi_{z}}, \Psi_{\mathrm{re}}\right), \\
\beta_{\mathrm{rest}}=-i \frac{\lambda}{\omega} \sum_{n} \lambda_{n s}^{\left(t_{1}\right)}\left(\frac{\Psi_{\mathrm{re}}}{\lambda-i \xi}, \Psi_{n t}\right)
\end{array}\right\} \quad\left(4.9^{\prime}\right)
$$

( 4.8$)$ は各 mode 間の関係式である。るし衝突がない時 $(\beta=0)$ は, 各 mode は number density の fluctuationa ${ }_{00}^{\vec{k}}$ だけに関係しているが, collision process のためK各 mode は coupleしているととが判る。

collision frequencyの一次まで考慮して dispersion relation を得るために (4.8)式で一度 iterationすると

$$
a_{\mathrm{re}}^{\overrightarrow{\mathbf{k}}}=\left[\alpha_{\mathrm{re}}+\sum \beta_{\mathrm{rest}} \alpha_{s t}\right] a_{0_{0}}^{\overrightarrow{\mathbf{k}}} \text {. }
$$

iteration が出来る条件, 又は（4、11）が正しく成り立つ条件壮，(4.8) の第2 項が 第 I 項に比して小さい場合である。

$$
\left|\frac{\beta}{\alpha}\right| \approx \frac{\lambda_{n s}^{(t)}}{\omega_{o}} \frac{k}{k_{D}} \ll 1 .
$$

従って(4、11)を用いて得られる dispersion relation は波数にがにっ比して小 さく, collision frequency が plasma frequency に比して小さい場合に正しい 
(4.11) で $\gamma=\ell=0$ とおくことにょって dispersion relation

$$
1=\alpha_{o o}+\sum_{\mathrm{re}} \beta_{o o r} \alpha_{\mathrm{re}}
$$

を得る。計算の便宜

$$
\mathbf{I}_{\mathbf{r e}} \equiv\left(\frac{i \xi_{z}}{\lambda-i \xi_{z}}, \Psi_{\mathbf{r} e}\right), \mathrm{J}_{\mathbf{r e}}=\left(\frac{1}{\lambda-i \xi}, \Psi_{\mathbf{r}-e}\right) \quad(4.14)
$$

とおくと

$$
\begin{aligned}
& I_{r e}=\lambda J_{r e}-\delta_{o r} \delta_{o e}, \\
& \alpha r e=-\left(\frac{\omega}{\omega}\right)^{2} 2 \lambda^{2} I_{r e} \\
& \beta_{o o r e}=-i \frac{\lambda}{\omega} \sum_{s} \lambda_{s \mathrm{r}}^{(e)} e_{s e}
\end{aligned}
$$

(4.13) $1\left(\frac{\omega}{\omega_{0}}\right)^{2}$ 加

$$
\left(\frac{\omega}{\omega_{0}}\right)^{2}=-2 \lambda^{2} \mathrm{I}_{o o}+i \frac{2 \lambda^{3}}{\omega} \sum_{\mathbf{r} s e} \lambda_{s \mathbf{r}}^{(\theta)} s_{s e} I_{r e}
$$

特に collisionless の場合は第 1 項だけ残り，(A、19)を用いて

$$
\left.\begin{array}{l}
\left(\frac{\omega}{\omega_{0}}\right)^{2}=1+3\left(\frac{\ell^{\prime}}{k_{\mathrm{D}}}\right)^{2}-i \frac{2 \gamma_{\mathrm{L}}}{\omega_{0}}, \\
r_{\mathrm{L}}=\sqrt{\frac{\pi}{8}} \omega_{0}\left(\frac{\ell_{\mathrm{D}}}{\ell_{2}}\right)^{3} \exp \left[-\frac{1}{2}\left(\frac{\ell^{2} \mathrm{D}}{\ell_{2}}\right)^{2}\right]
\end{array}\right\}
$$

てれは良く知られた分散式である。 $\gamma_{L}$ は Landau damping係数を意味する。

電子一電子 collision の際の collision invariance 加

$$
\lambda_{\mathrm{r} o}^{(o)}=\lambda_{\mathrm{r}_{1}}^{(0)}=\lambda_{\mathrm{r} 0}^{(0)}=0, \quad \text { for } \mathrm{r}=0,1,2, \cdots . \quad(2,18)
$$


従って $\sum_{\mathbf{r}}^{\infty} \sum_{=0}^{\infty}$ の内 $\left(\begin{array}{c}s \\ \mathbf{r}\end{array}, \ell\right)=(0,0),(1,0),(0,1)$ 《落ち百。従って $(4$ 。 $16)$ は (4.15)を用的て

$$
\left(\frac{\omega}{\omega_{0}}\right)^{2}=-2 \lambda^{2} \mathbf{I}_{o o}+i \frac{2 \lambda^{4}}{\omega} \sum_{s \mathrm{r} e}^{\prime} \lambda_{\mathrm{r} s}^{\ell)} J_{s e} J_{\mathrm{re}} . \quad(4.19)
$$

こてに primeは collision invarianceのために寄与しない項を除くことを意味する。

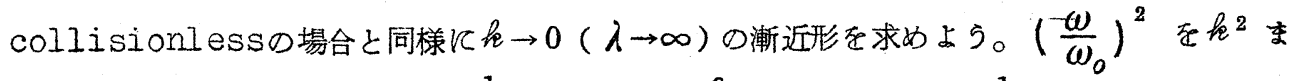

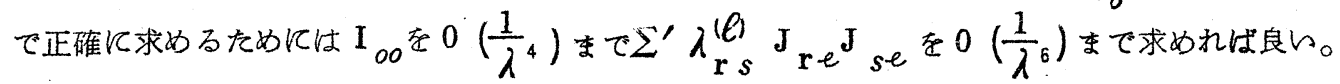
(4.14)を用いて

$$
J_{s \ell}=\frac{1}{\lambda}\left(1, \Psi_{s e}\right)+\frac{i}{\lambda^{2}}\left(\xi_{z}, \Psi_{s e}\right)-\frac{1}{\lambda^{3}}\left(\xi_{z}^{2}, \Psi_{s e}\right) \cdots(4,20)
$$

一方漸化式 $(\mathrm{B} \cdot 6)$ :

$$
\xi_{z} \Psi_{\mathrm{r} e}=\mathrm{A}_{\mathrm{r} e} \Psi_{\mathrm{r}, \ell+1}-\mathrm{B}_{\mathrm{r} e} \Psi_{\mathrm{r}-1, \ell+1}+\mathrm{C}_{\mathrm{r} e} \Psi_{\mathrm{r}, \ell-1}-\mathrm{D}_{\mathrm{r} e} \Psi_{\mathrm{r}+1, \ell-1}
$$

を考えると， $\xi_{z}^{n}$ は $\Psi_{o 0}=1$ K $\xi_{z}$ を回 operate したすのと解釈出来る。すると $\xi_{z}^{n}$

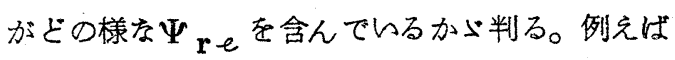
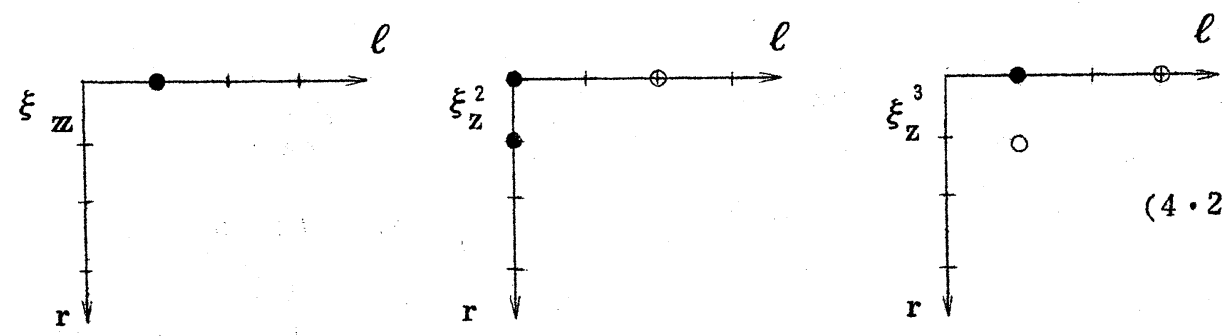

○と・の位置にそれぞれの場合 $\mathbf{r} \boldsymbol{r}$ が現われる。・は collision invariance (4・18)

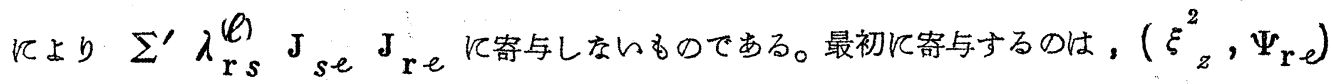
の内 $(\stackrel{r}{s}, \ell)=(0,2)$ であるととが $(4.21)$ 図から判る。此の様にして

$$
\sum_{\mathrm{r} s e}^{\prime} \lambda_{\mathrm{r} s}^{(\varphi)} J_{s e} J_{\mathrm{re}}=\frac{\lambda_{o 0}^{(2)}}{3} \frac{1}{\lambda^{6}}+0\left(\frac{1}{\left.\lambda^{8}\right)}\right.
$$


を得る。こてでの奇数巾が出ないてとは次の様に説明出来る。 $\left(\xi_{z}^{n} \Psi_{\mathrm{re}}\right)$ の内 $n$ が偶 (奇)

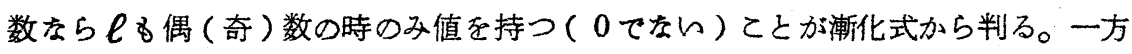

$$
J_{s e} J_{\mathbf{r e}}=\frac{1}{\lambda^{2}} \sum_{n_{m}}\left(\left[\frac{i \xi_{z}}{\lambda}\right]^{n}, \Psi_{s e}\right) \cdot\left(\left[\frac{i \xi_{z}}{\lambda}\right]^{m}, \Psi_{\mathbf{r e}}\right),
$$

従って $\ell$ 二偶 (奇) 数ならば $n$ むる 同時に偶 (奇) 数の時のみ $\sum n_{m}$ に奇与する。故に $n+m$ は常に偶数になる。

(A. 19 ) 加

$$
-2 \lambda^{2} I_{o o}=1-\frac{3}{2 \lambda^{2}}+\cdots,
$$

$(4 \cdot 22)(4 \cdot 23)$ を( $4 \cdot 19)$ に代入して

$$
\left(\frac{\omega}{\omega_{0}}\right)^{2}=1+3\left(\frac{k}{k \mathrm{D}}\right)^{2}-i \frac{4}{3} \frac{\lambda_{00}^{(2)}}{\omega_{0}}\left(\frac{k}{k_{\mathrm{D}}}\right)^{2}
$$

乙の dispersion relation は

$$
\text { collision frequency }<\text { plasma frequency }
$$

の限り正しh。

以上では collision frequency の一次迄しか扱わなかったが，(4・8).式でn回 iteration するととにより collision frequency のn次の項からの dispersion relation への寄与を前と同様にして計算出来る。従って collision frequency $の$ 任意次数まで考虑した dispersion relation が求められる。n回 iteration する 時, $\beta$ の 乗の項は

$$
\mathrm{K}_{n}=\mathrm{r}_{1} \ell_{1} \mathbf{r}_{2} \ell_{2} \ldots \mathrm{r}_{n} \ell_{n}=0{ }^{\beta}{ }_{\text {oor } \mathrm{r}_{1} \ell_{1}} \beta_{\mathbf{r}_{1} \ell_{1} \mathrm{r}_{2} \ell_{2}}^{\beta}{ }_{\mathbf{r}_{n-1}} \ell_{n-1} \mathbf{r}_{n} \ell_{n}^{\alpha} \mathbf{r}_{n} \ell_{n}
$$

だけ dispersion relation に奇与する。そして dispersion relationは

$$
1=\alpha_{o \circ}+\sum_{n=1}^{\infty} \mathrm{K}_{n}
$$

となる。（4.13）壮此の式で $n=1$ とおりたすのである。 $\mathrm{K}_{n}$ は $n$ 個の $\beta$ と 1 個の $\propto$ と積 てあるが, 各 $\alpha, \beta$ の $\frac{1}{\lambda}$ 展開に於ける最初の項同志の積が, $\mathrm{K}_{n}$ の $\frac{1}{\lambda}$ 展開於ける $0\left(\frac{1}{\lambda^{2}}\right)$ 
小笠原

の項を与えるととを以下で示そう。

定義（4、9'）を用的て

$$
\begin{aligned}
& \beta_{o o r_{1} \ell_{1}}=-i \frac{\omega_{o}}{\omega} \lambda \sum_{\mathrm{s}_{1}} \frac{\lambda_{\mathrm{s}_{1} \mathrm{r}_{1}}^{\left(\ell_{1}\right)}}{\omega_{0}}\left(\frac{1}{\lambda-i \xi_{z}}, \Psi_{\mathrm{s}_{1} \ell_{1}}\right), \\
& \beta_{\mathrm{r}_{i-1} \ell_{i-1} \mathbf{r}_{i} \ell_{i}}=-i \frac{\omega_{0}}{\omega} \lambda \sum_{s_{i}} \frac{\lambda_{\mathrm{s}_{i} \mathrm{r}_{i}}^{\left(\ell_{i}\right)}}{\omega_{o}}\left(\frac{\Psi_{\mathbf{r}_{i-1} \ell_{i}-1}}{\lambda-i \xi_{z}}, \Psi_{\mathrm{s}_{i} \ell_{i}}\right), \\
& \alpha_{\mathrm{r}_{n} \ell_{n}}=-\left(\frac{\omega_{o}}{\omega}\right)^{2} 2 \lambda^{2}\left(\frac{i \xi_{z}}{\lambda-i \xi_{z}}, \Psi_{\left.\mathrm{r}_{n} \ell_{n}\right)}\right.
\end{aligned}
$$

$(4 \cdot 20)$ から $(4 \cdot 22)$ までの議論に従うと

$$
\begin{aligned}
& \beta_{o o \mathbf{r}_{1} \ell_{1}}=-\frac{\omega_{0}}{\omega}\left(\frac{-i \lambda_{o \mathbf{r}_{1}}^{(2)}}{\omega_{o}}\right) \frac{1}{\sqrt{3} \lambda^{2}} \delta_{2 \ell_{1}}+o\left(\frac{1}{\lambda^{3}}\right), \\
& \beta_{\mathbf{r}_{i-1} \ell_{i-1} \mathbf{r}_{i} \ell_{i}}=\frac{\omega_{0}}{\omega}\left(\frac{-i \lambda_{\mathbf{r}_{i-1} \mathbf{r}_{i}}}{\omega_{0}}\right) \delta_{\ell_{i-1}} \ell_{i}+0\left(\frac{1}{\lambda}\right),(4.27)
\end{aligned}
$$

$$
\alpha_{\mathbf{r}_{n} \ell_{n}}=\left(\frac{\omega_{o}}{\omega}\right)^{2} \frac{2}{\sqrt{3}} \delta_{o \mathbf{r}_{n} \delta_{2 n}}+0\left(\frac{1}{\lambda}\right) .
$$

これらを(4.25)に代入すると，

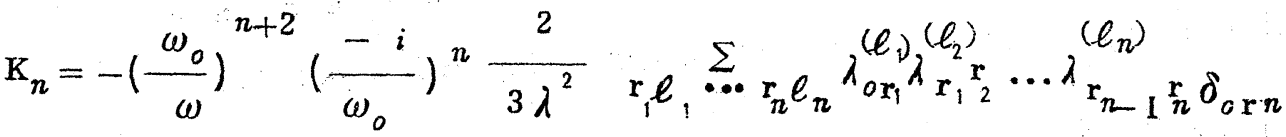

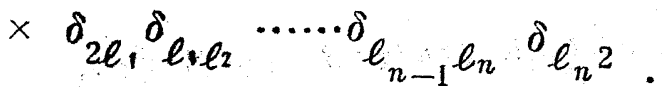

132 
徉。 $\quad \mathrm{K}_{n}=-\frac{2}{3 \lambda^{2}}\left(\frac{-i}{\omega_{0}}\right)^{n}\left(\frac{\omega_{0}}{\omega}\right)^{n+2}\left(\lambda^{Q) n}\right]_{00}, \quad(4.28)$

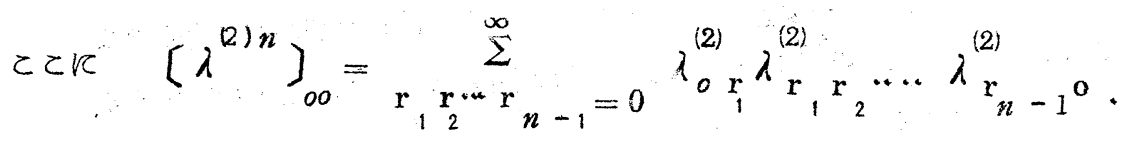

(4.28)を( 4.26$)$ 飞代入し $(4.26) \times\left(\frac{\omega}{\omega_{0}}\right)^{2}$ 加

$$
\left(\frac{\omega}{\omega_{0}}\right)^{2}=-2 \lambda^{2} \mathrm{I}_{o o}-\sum_{n=1}^{\infty}\left(\frac{\omega_{0}}{\omega}\right)^{n}\left(\frac{-i}{\omega_{0}}\right)\left[\lambda^{(2) n}\right]_{00} \frac{2}{3 \lambda^{2}}
$$

(4.23)を用いて

$$
\left(\frac{\omega}{\omega_{0}}\right)^{2}=1+3\left(\frac{h_{\ell}}{h_{D}}\right)^{2}+\frac{4}{3}\left(\frac{h}{h_{D}}\right)^{2} \sum_{n=1}^{\infty}\left(\frac{-i}{\omega_{0}}\right)^{n}\left[\lambda^{(2)}\right]_{00}(4.30)
$$

$\lambda_{\mathrm{pq}}^{(2)}$ の值はIで計算されており

$$
n_{0} \overline{\mathrm{K}}=\left(\frac{\mathrm{e}^{2}}{\kappa \mathrm{T}_{0}}\right)^{2}\left(\frac{\pi \kappa \mathrm{T}_{0}}{m}\right)^{1 / 2} \ln \left(\frac{\kappa \mathrm{T}_{0} \ell_{\mathrm{D}}}{\mathrm{e}^{2}}\right) n_{0} \quad(4.31)
$$

を単位にして係数だけの表が与えられている。ての係数 matrixをB $\mathbf{p ~ q}$ と書くことにする。 この数值を用いて次に $\left[\lambda^{(2)} n\right]$ 在計算する。 matrixßの固有值問題が解けていると

$$
\mathrm{LB} \mathrm{L} \mathrm{L}^{-1}=\left[\begin{array}{lllll}
\lambda_{0} & \lambda_{1} & & & \\
& \lambda_{1} & \lambda_{2} & & 0 \\
& 0 & & \ddots & \ddots
\end{array}\right]
$$

を满たす㨁交変換Lと固有值入; 功存在する。

その時

となり

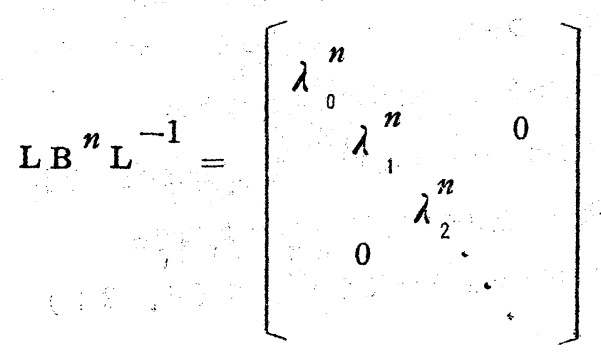




$$
\mathrm{B}_{o 0}^{n}=\sum_{i=0}^{\infty} \mathrm{L}_{i 0}^{2} \lambda_{i}^{n}=\frac{\left[\lambda^{(2) n}\right]_{00}}{\left(n_{0} \overline{\mathrm{K}}\right)^{n}}
$$

と書ける。ところがB壮無限次元なのて，有限次元て cutして固有值問題を解かね枝ならなな

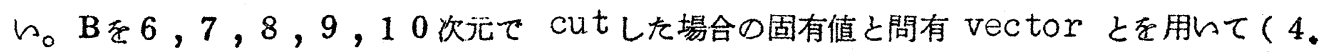
32 ) を計算すると次表のよらになる。

\begin{tabular}{c|ccccc} 
次元 $\rightarrow$ & 6 & 7 & 8 & 9 & 10 \\
\hline$\sum L_{i 0}^{2} \lambda_{i}^{2}$ & 3.0592 & 3.0593 & 3.0593 & 3.0594 & 3.0594 \\
$\sum L_{i 0}^{3} \lambda_{i}^{3}$ & 7.4058 & 7.4155 & 7.4182 & 7.4186 & 7.4189 \\
$\sum L_{i 0}^{4} \lambda_{i}^{4}$ & 24.533 & 24.884 & 25.042 & 25.106 & 25.137 \\
$\sum L_{i 0}^{5} \lambda_{i}^{5}$ & 114.72 & 122.75 & 127.57 & 130.30 & 131.85 \\
\hline
\end{tabular}

従って

$$
\begin{aligned}
& \lambda_{00}^{(2)}=1.6 n_{0} \overline{\mathbf{K}} \\
& {\left[\lambda^{(2) 2}\right]_{00}=3.0595\left(n_{0} \overline{\mathrm{K}}\right)^{2}(2.56)} \\
& {\left[\lambda^{(2) 3}\right]_{00}=7 \cdot 42\left(n_{0} \overline{\mathrm{K}}\right)^{3}} \\
& \text { (4.096) } \\
& \text { (4. } 33 \text { ) } \\
& {\left[\lambda^{(2) 4}\right]_{00}=25.2\left(n_{0} \bar{K}\right)^{4}} \\
& \text { (6.56) }
\end{aligned}
$$

$\S 5$ Discussion of the Dispersion Relation

前節の結果，電子一電子衝突の影響は $\ell=2 の$ mode のみであるととが判った。一般にイオ ン一電子衝笑を考慮してもO $\left(k^{2}\right)$ まで考える限り， $\ell>2 の$ modeは現われない。

Johnston は全然説明なしK $\ell>2$ を始めから考えないて conductivity tensor を計 算しているが，我々の結果からするとJohnston の扱いは結果的に正しかった事が判る。

分散式の意味簡単に調へ上う。collision firequencyの一次迄採用して (4.24) か5 


$$
\omega=\omega_{0}+\frac{3}{2} \omega_{0}\left(\frac{k}{k_{D}}\right)^{2}-i \frac{2}{3} \lambda_{o 0}^{(2)}\left(\frac{h}{k_{D}}\right)^{2}
$$

$\lambda_{\text {O。 }}^{(2)}$ は縦，横の温度が異なるいわゆる楕円分布：

$$
f\left(v_{11}, v_{\perp}\right) \infty \exp \left[-\frac{v_{11}^{2}}{2 \kappa \mathrm{T}_{11}}-\frac{v_{\perp}^{2}}{2 \kappa \mathrm{T}_{\perp}}\right]
$$

の maxwellization rate, 又山両温度間の rate of relaxation

$$
\frac{d}{d t}\left(\mathrm{~T}_{\perp}-\mathrm{T}_{11}\right)=-\lambda_{o 0}^{(2)}\left(\mathrm{T}_{\perp}-\mathrm{T}_{11}\right)
$$

を与える。（5、1）は又

$$
\frac{\partial n}{\partial t}=-i \omega_{o} n+i \frac{3}{2} \frac{x \mathrm{~T}_{o}}{m} \frac{1}{\omega_{0}} \frac{\partial^{2} n}{\partial \mathrm{z}^{2}}+\frac{2}{3} \frac{\lambda_{o 0}^{(2)}}{\ell_{\mathrm{D}}^{2}} \frac{\partial^{2} n}{\partial \mathrm{z}^{2}} \quad(5,2)
$$

なる方程式からす導かれる。右辺第 1 項は plasma oscillation 第 2 項柱分散項を与え

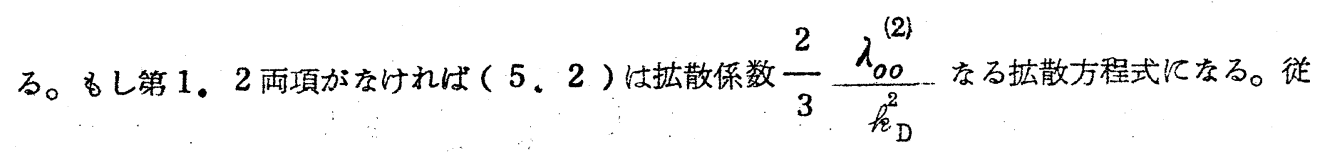
って我々の扱っている振動は number densityの変動によって生ずる charge を消す振動 $\left(\omega_{0}\right)$ と共に，その時生ずる縦，横の温度差を消す様に払散しているととが判る。又 collisionK比して影響は小さいが, potential trough K trap された粒子と共鳴 的に作用して波怙減衰する。（Landau damping）

我々の結果と比較し得方他の結果怄, Bhatnager, Gross and Krook ${ }^{7}$ ) ( B-G-K) のるののみである。その他のるのは collision invariance を考慮していないからでる。 B-G-K仕 single collision frequency model から出発し

$$
\frac{\omega}{\omega_{0}}=1+\frac{1}{2}\left(\frac{k}{k_{D}}\right)^{2} \frac{1}{\omega_{0}^{2}+\lambda^{2}}\left[\left(3 \omega_{0}^{2}+\frac{5}{3} \lambda^{2}\right)-i \frac{4}{3} \omega_{0} \lambda\right](5,3)
$$

なる dispersion relation を与えてる。

彼等はどんな mode の分布子皆同一緩和時間を持つと仅定している。そのために計算は 
小笠 原

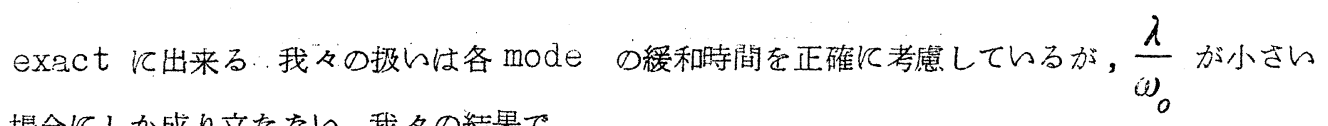
場合にしか成り立たない。我々の結果で

$$
\lambda_{\mathrm{pq}}^{(2)}=\lambda \text { for all pøg }
$$

とすると当然のととながら（ 4 ・30)と（5.3）は一致する。次に single relaxation constant theory がぞの程度の近似になっているかを兒るために 4 、 33 ) において

$$
\lambda_{o o}^{(2)}=1 \cdot 6 n_{o} \overline{\mathrm{K}} \equiv \lambda
$$

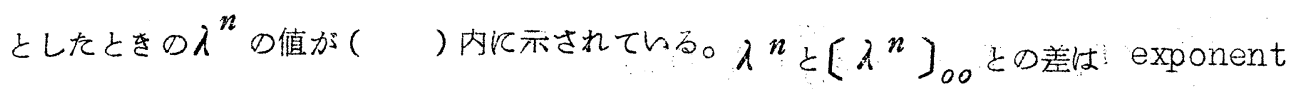
nが大さくなるつれて非常に大をくなる。従って B-G-Kの model は collision frequency の高い次数まで考える場合は余り良くないてとが判る。（4。30）で

$$
\omega=\omega_{\mathbf{r}}-i \omega_{i},\left|\frac{\omega_{i}}{\omega \mathbf{r}}\right| \ll 1
$$

とおくと

$$
\begin{aligned}
& \left(\frac{\omega_{\mathbf{r}}}{\omega_{0}}\right)^{2}=1+3\left(\frac{h^{2}}{\ell_{D}}\right)^{2}\left[1-\frac{4}{9}\left[\frac{\lambda^{(2) 2}}{\omega_{0}}\right]_{00}+\frac{4}{9}\left[\frac{\lambda^{(2) 4}}{\omega_{0}}\right]_{\infty} \ldots \ldots\right] \\
& (5,4) \\
& \omega_{i}=\frac{2}{3} \omega_{o}\left(\frac{\ell_{0}}{k_{D}}\right){ }^{2}\left[\frac{\lambda_{o 0}^{(2)}}{\omega_{0}}-\left[\frac{\lambda^{(2) 3}}{\omega_{0}}\right]_{00}+\ldots \ldots . . .\right],(5.5)
\end{aligned}
$$

この様に collision frequency は $\omega_{\mathrm{r}}$ 壮偶数巾で, $\omega_{i}$ Vは奇数巾で入 て来る。

良く用いられる plasma parameter g は Đebye length を半径として持つ球 中の粒子数の逆数で定義され，通常非常に小さい值を持つ。

$$
g=\left(\frac{\mathrm{e}^{2}}{\kappa \mathrm{T}_{\mathrm{D}}}\right)^{3 / 2} n_{0}^{\mathrm{t} / 2} \ll 1
$$


collision integral (collision Prequency) $\lambda_{\mathrm{pq}}$ Ф計算水的て impact parameterが大さい所で発散する。それを防ぐために impact parameter = Debye lengthめ所で cut off する。そ心際

$$
\ell n\left(\frac{1}{g}\right) \gg 1
$$

なるととを用いて, 入 $\mathrm{pq}$ は一般に

$$
\lambda_{\mathbf{p} q}=\mathbf{A}_{\mathbf{p} q} \omega_{o} g \ell_{n g},
$$

$$
A_{\text {p } q}=\text { 数係数 }
$$

と書ける。従って分散式に Collision の効果俚

$$
\begin{aligned}
& \left(\frac{\lambda}{\omega_{o}}\right)^{n}=\mathrm{L}_{n}(g \ell n g)^{n}, \\
& \mathrm{~L}_{n}=\text { 数係数 }
\end{aligned}
$$

の形で入って来る。

以上は Boltzmann-VIasov eg。を基礎方程式とした議論であるが, plasma の 基碟方程式として最近，多体相関考考慮した kinetic eq。老作る研究が战されている ${ }^{3}$ ) Ichikawa $\left.{ }^{4}\right)$, Willis ${ }^{5}$ ) 5は此の方法で plasma oscillationt扱っている。彼 等壮 3 体分布函数を 2 体と 1 体との積で近似するととにより，階級方程式をclose させている。 Willis は $\frac{\partial f_{1}}{\partial t}$ K対す㟢与を波数 $\overrightarrow{\mathbf{k}}$ Kindependent项(A)と dependent とに分けている。

$$
\begin{aligned}
& \frac{\partial f_{1}}{\partial t}=(\mathbf{A}), \\
& \frac{\partial f_{1}}{\partial t}+i \overrightarrow{\mathbf{k}} \cdot \vec{v} f_{1}+\left(\mathbf{S}_{0} \mathbf{C}, \mathbf{F}\right)=(\mathbf{B}) .
\end{aligned}
$$

ここにf、は1体分布函数の平衡からのはずれを意昧する。（S、C、F、）は self consistent field 関係した項である。ionは positive charge back: ground としてのみ作用している。 $(5,10)$ は collision term に対応していて 


$$
\text { 小笠原 }
$$

Fokker-Planck type に書かれる。その係数は 2 体 correlation 効果のために発散 なしに求まる。我々は

$$
\frac{\partial f_{1}}{\partial t}+i \vec{k} \cdot \vec{v} f_{1}+\left(S_{0} C_{2} \mathbf{F} .\right)=(\mathbf{A})
$$

を解らたととになる。illisは（5，11）を基礎方程式として

$$
\begin{aligned}
& \left(\frac{\omega_{\mathbf{r}}}{\omega_{o}}\right)^{2}=1+3\left(\frac{\ell^{\prime}}{\ell_{D}}\right)^{2}\left[\begin{array}{c}
9.2 \mathrm{~g}] \\
(1.05)
\end{array}\right. \\
& \omega_{i}=17.6 \omega_{0} g\left(\frac{\ell^{2}}{(0.03)}\right)^{2}
\end{aligned}
$$

を導らている。（）内の数値は Ichikawa の結果である。我々の結果からすると $\left(\frac{\omega_{\mathbf{r}}}{\omega_{0}}\right)^{2}$ の dispersive term のgの ordero項は出ない。従って此の項は 2 体相関 に特有の項である。多体相関効果を詳しく調へることは，今後の大きな課題の一つであろう。

$\$ 6$ Integrated Noment Equation

今迄は Boltzmann-Vlasov eqを $(4,7)$ の5に変形してから momentを求めた が,此の section で普通やられるよらに，そのままの形で momentを採り方程式系を導 く。それらを integrated moment eg。と名づける。とれらの方程式系を用いて現存す る理論と，我々の前節迄の結果につんて簡単に調へる。

$$
(4,3) \text { を Fourier 変換して }(4,6) \text { を用的ると }
$$

$\frac{\partial \phi_{k}}{\partial t}+i \operatorname{lov}_{\mathrm{z}} \phi+\sqrt{2 \alpha} \omega_{0} \frac{i h_{\mathrm{D}}}{\ell} v_{\mathrm{z}} a_{o 0}^{\ell}=-n_{0} \mathrm{~J} \phi_{k}$.

展開 (2.8) を用いて

$$
\begin{gathered}
\sum \dot{a}_{i j} \Psi_{i j}+i k \sum_{i j} a_{i j}^{k} v_{\mathrm{z}} \Psi_{i j}+\sqrt{2} \alpha \omega_{0} \frac{i h_{\mathrm{D}}}{k} v_{\mathrm{z}} \dot{a}_{o o}^{k} \\
=-\sum_{i j \mathrm{~s}} \lambda_{i \mathrm{~J} \mathrm{~s}}^{(j)} a_{i j}^{k} \Psi_{\mathrm{s} j} .
\end{gathered}
$$


Moment Method for Wave Phenomena in Plasma

この両辺に $d \overrightarrow{\mathrm{v}} f^{(0)} \Psi_{\mathbf{r}_{e}}$ をoperate すると

$$
\begin{aligned}
\dot{a}_{\mathbf{r}_{e}}^{k} & +\frac{i k}{\alpha} \sum_{i j} a_{i_{j}}^{k}\left(\Psi_{\mathbf{r}_{e}}, \xi_{\mathrm{z}} \Psi_{i j}\right)+\sqrt{2} \frac{i k_{\mathrm{D}}}{h}\left(\Psi_{\mathrm{r}_{e}}, \xi_{\mathrm{z}}\right) a_{o o}^{k} \\
& =-\sum_{i} \lambda_{\mathrm{ri}}^{())} a_{i_{e}}^{k} .
\end{aligned}
$$

漸化式 $(\mathrm{B} ・ 6)$ を用いて第 2 項を変形すると

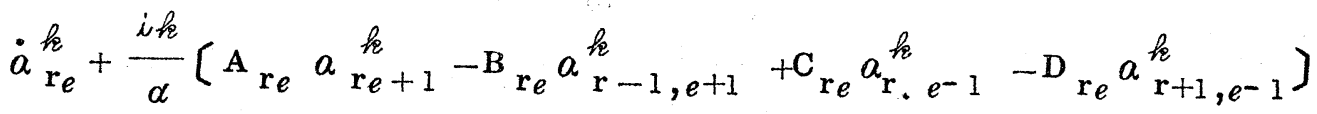

$$
\begin{aligned}
& +i \omega_{0} \frac{k_{\mathrm{D}}}{k} a_{o o}^{k} \delta_{\text {or }} \delta_{1 e}=-\sum_{i} \lambda_{\mathrm{ri}}^{(\ell)} a_{i_{e}}^{k_{e}} \quad(6,1)
\end{aligned}
$$

を得る。こてで係数 $\mathrm{A} \mathrm{r}_{e}, \mathrm{~B}_{\mathrm{r}_{e}}, \cdots \cdots$ 等の数值什（ $\mathrm{B}, 7$ ）で支えられるるのである。

(6.1) の最初の3つは

$$
\begin{aligned}
& (\mathbf{r}, e) \\
& (0,0): \dot{a}_{o 0}^{k}+i \omega_{0} \frac{k}{k_{D}} a_{o, 1}^{k}=0, \\
& (0,1): \dot{a}_{o 1}^{k}+i \omega_{0} \frac{k}{k_{D}}\left[a_{o O}^{k}-\sqrt{\frac{2}{3}} a_{10}^{k}+\frac{2}{\sqrt{3}} a_{o_{2}}^{k}\right] \\
& +i \omega_{o} \frac{k_{D}}{k} a_{o 0}^{k}=0 \\
& (1,0): \dot{a}_{10}^{k}+i \omega_{0} \frac{k}{k_{D}}\left[\sqrt{\frac{5}{3}} a_{11}^{k}-\sqrt{\frac{2}{3}} a_{01}^{k}\right]=0 .
\end{aligned}
$$

これらに（2，19)を代入すると linearize されを連続の式,運動方程式, energy の 式が得られる。（ $6 ， 1)$ の係数を表にすると次の上5になる。 
小笠原

\begin{tabular}{|c|c|c|c|c|c|c|}
\hline & $a_{0 o}$ & $a_{01}$ & $a_{10}$ & $a_{02}$ & $a_{12}$ & $a_{22}$ \\
\hline$a_{00}$ & $\omega$ & $-\omega_{0} x$ & 0 & 0 & $\because \quad 0$ & 0 \\
\hline$a_{01}$ & $-\frac{\omega_{0}}{x}\left(1+x^{2}\right)$ & $\omega$ & $\omega_{0} \frac{\sqrt{6}}{3} x$ & $-\omega_{0} \frac{\sqrt{12}}{3} x$ & 0 & 0 \\
\hline$a_{10}$ & 0 & $\omega_{o} \frac{\sqrt{6}}{3} x$ & $\omega$ & 0 & 0 & 0 \\
\hline$a_{02}$ & 0 & $-\omega_{0} \frac{\sqrt{12}}{3} x$ & 0 & $\omega+\Lambda \circ$ & $\therefore \quad \Lambda_{01}$ & $\Lambda_{02}$ \\
\hline$a_{12}$ & 0 & 0 & 0 & $\Lambda_{10}$ & $\omega+A_{11}$ & $\Lambda_{12}$ \\
\hline$a_{22}$ & 0 & 0 & 0 & $\Lambda_{20}$ & $\Lambda_{21}$ & $\omega+\Lambda_{22}$ \\
\hline
\end{tabular}

$$
x \equiv \frac{h^{k}}{h_{D}}, \quad \Lambda_{i j} \equiv i \lambda_{i j}^{(2)} .
$$

てれを適当な次元て truncate するととにより種々のdispersion relation を得る ことが出来る。 Matrixの配列の仕方は $2 \mathrm{r}+\ell$ の值が増すようになっている。 (polynomial ordering) $2 \mathbf{r}+\ell$ は各 moinent の次数 $\left(v^{2} \mathbf{r}+\ell\right)$ を意味する。 Thomson ") eg。を用いて扱っている。その後 Bailey $\left.{ }^{1^{2}}\right)$ はeq。 of continuity, nomentum, Poisson'seg.を用唒

$$
\omega^{2}=\omega_{0}^{2}+\frac{\kappa T_{0}}{m} \ell^{2}
$$

を与えている。この結果は上の matrix で 2 次元まで採ると得られる。3 次元迄採ると 
Moment Method for Wave Phenomena in a Plasma

$$
\omega^{2}=\omega_{o}^{2}+\frac{5}{3} \quad \frac{\kappa \mathrm{T}_{o}}{m} k^{2}
$$

となり，乙れは断熱変化の式を用いた場合と同じ結果を与える。乙の場合は Gross

13) KL って扱われている。3 次元交で考虑するてとは, $a_{\text {oo }}, a_{o 1}, a_{10}$ のつの moment のみ が存在する場合を考慮しているとと火なる。てれらのmoment は collision invariance のために collision dampingをしない。他の mode は exciteされたとしてもhigh density の場合すぐ dampして了う。従って鼠初の3つの momentのみ考虑するととは， high density case 考えているてとになる俰数 $\frac{5}{3}$ について以上のように解釈される のである。

次壮 stress 考慮して，4次元迄採ると

$$
\omega^{2}=\omega_{0}^{2}+3 \frac{\kappa \mathbf{T}_{0}}{m} k^{2}
$$

となり, 前々節で得られたものと一致する。てれは Iow density の場合 exact な結果に なっている。表には書いてないが, $a_{11}, a_{03}, \cdots \cdots$ 等を考えて $5,6 \cdots \ldots$ 次元迄採っ てもO $\left(\ell^{2}\right)$ までは $(6,4)$ が成り立つ, 但し4次元以上は collisionは考えない場合に ついてである。今 $2 \mathbf{r}+\ell$, 即ち各 moment が velocityの何次の momentになるかを示 するのを書くと

\begin{tabular}{|r|c|c|c|c|c|c|}
\hline$a_{\mathrm{r} \ell}$ & $a_{00}$ & $a_{01}$ & $a_{10}$ & $a_{02}$ & $a_{11}$ & $a_{03}$ \\
\hline $2 \mathrm{r}+\ell$ & 0 & 1 & 2 & 2 & 3 & 3 \\
\hline
\end{tabular}

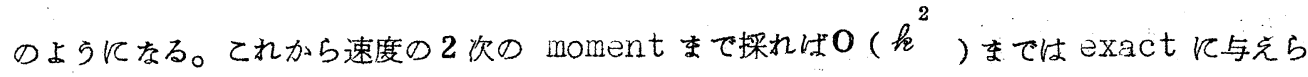
れると云える。又は 3 次以上の momen妒 $0\left(\ell^{2}\right)$ までに寄与しないとす云える。従って $\omega^{2}$ の $\ell^{2}$ の項まで求めるために $\ell=2$ 以下の mode のみを考慮した Johnstonの扱いは正しい ととが判る。

integrated moment method で collisionが存在する場合を扱い，§4で得た 結果と比較する。基礎方程式は $a_{00}, a_{01}, a_{10}, a_{02}$ 亿対する4つで良い筈であるが，（6。 1 ）を用いて $a_{02}$ 関する式を書くと 


$$
\begin{gathered}
\text { 小 笠 原 } \\
\dot{a}_{02}^{k}+i \omega_{0} \frac{k}{k_{D}} a_{01}^{k}=-\sum_{n=0}^{\infty} \lambda_{o n}^{(2)} a_{n_{2}}^{k} \quad(6,5)
\end{gathered}
$$

となり，無限に多くの momentが関与してくる。てのためにa ${ }_{n_{2}}$ に対する方程式も考慮しな ければならない。最初第 1 近似として，（6、5）の右辺で $n=0$ のみを考えることにすると， 表に於いて4 次元迄採ったととになる。その場合 dispersion relation は

$$
\left(\omega+i \lambda_{o O}^{(2)}\right)\left(\omega^{2}-\omega_{o}^{2}-\frac{5}{3} \omega_{o}^{2} \frac{k^{2}}{k_{D}^{2}}\right)-\omega \omega_{o}^{2} \frac{4}{3}\left(\frac{k}{k_{D}}\right)^{2}=0
$$

となる。てれから

$$
\begin{gathered}
\lambda_{o 0}^{(2)} \gg|\omega| \cdots \cdots \text { high density } \\
\omega^{2}=\omega_{0}^{2}+\frac{5}{3} \omega_{0}^{2}\left(\frac{k}{k_{D}}\right)^{2} . \\
\lambda_{00}^{(2)} \ll \omega \quad \cdots \cdots \text { low density } \\
\omega^{2}=\omega_{0}^{2}+3 \omega_{0}^{2}\left(\frac{k^{2}}{k_{D}}\right)^{2}
\end{gathered}
$$

となる。るっと一般的に解くと

$$
\begin{aligned}
& \omega_{\mathbf{r}}^{2}=\omega_{o}^{2}+3 \omega_{0}^{2}\left(\frac{k}{k_{D}}\right)^{2}, \\
& \omega_{i}=\frac{2}{3} \lambda_{o 0}^{(2)}\left(\frac{h^{2}}{h_{D}}\right)^{2}
\end{aligned}
$$

となり，(4.24)と同じ結果になる。

第 2 近似（6、5)で $n=0 、 1$ を採用する。

表で 5 次元まで採るととになる。前と同様にして

$$
\left(\frac{\omega}{\omega_{0}}\right)^{2}=1+3\left(\frac{k}{k_{\mathrm{D}}}\right)^{2}+\frac{4}{3}\left(\frac{k}{h_{\mathrm{D}}}\right)^{2} \sum_{n=1}^{2}\left(\frac{-i}{\omega_{0}}\right)^{n}\left[\lambda^{(2) n}\right]_{0 o}^{\prime}(6,6)
$$

乙टK 


$$
\left[\lambda^{(2) 2}\right]_{00}^{\prime}=\sum_{i=0}^{1} \lambda_{o i}^{(2) 2},\left[\lambda^{(2) 1}\right]_{00}^{\prime}=\lambda_{o 0}^{(2)} .
$$

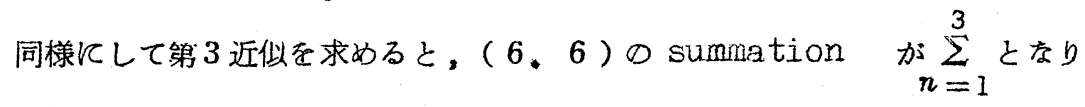

$$
\left[\lambda^{(2) 3}\right]_{o o}^{\prime}=\sum_{i, j=0}^{2} \lambda_{o i}^{(2)} \lambda_{i j}^{(2)} \lambda_{j o}^{(2)}
$$

となるだけで他は同じである。一般に第 $\mathbf{r}$ 近似をすると

$$
\begin{aligned}
& \left(\frac{\omega}{\omega_{0}}\right)^{2}=1+3\left(\frac{k}{k_{\mathrm{D}}}\right)^{2}+\frac{4}{3}\left(\frac{k}{k_{\mathrm{D}}}\right)^{2} \sum_{n=1}^{\mathrm{r}}\left(\frac{-i}{\omega_{0}}\right)^{n}\left[\lambda^{(2)}\right]_{00}^{\prime},(6,8) \\
& {\left[\lambda^{(2) n}\right]_{00}^{\prime}=\sum_{\mathrm{s}_{1} \mathrm{~s}_{2} \cdots \mathrm{s}_{n-1}=0}^{\mathrm{r}}{ }^{-1} \lambda_{\mathrm{os}_{1}}^{(2)} \lambda_{\mathrm{s}_{1} \mathrm{~s}_{2} \ldots \lambda_{\mathrm{s}_{n-1}}^{(2)} \ldots}^{(2)}(6,9)}
\end{aligned}
$$

となる。( $6 、 8)$ と $(4 、 30)$ 壮等しい形である。しかし $(6 、 9)$ は $(4.29)$ と少し 異なる。しかしながら $\mathrm{r} \rightarrow \infty$ の時，\$4の結果と本節の結果は一致する。此の様にして我々が考 えている振動に関与する moment は

$$
a_{00}, a_{01}, a_{10}, a_{i_{2}}, i=0,1,2 \ldots \ldots
$$

であるととが判る。

\section{\$7. Moment Wave}

(4.8)により種々の moment の間の関係が与えられる。特に collision を考えな い場合には

$$
a_{\mathbf{r}_{e}}^{k}=\alpha \mathbf{r}_{e} a_{o o}^{k}
$$

となり全ての mode壮 number density のfluctuation で表わせることが判る。実

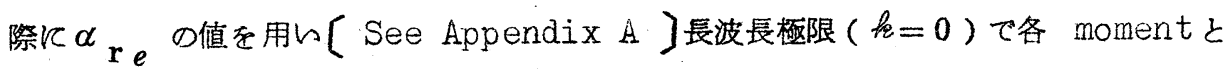
number density fluctuation とがどの上うに関係しているが兒る。

( 2. 19$),(7.1)$, dispersion relation $\alpha r_{e}$ の值を用饭

$$
\begin{aligned}
& E \equiv \exp (i \overrightarrow{\mathbf{k}} \cdot \overrightarrow{\mathbf{x}}-i \omega t), \\
& \widetilde{n}^{k}=a_{o o}^{k} \mathrm{E},
\end{aligned}
$$




$$
\begin{aligned}
& \text { 小 笠 原 }
\end{aligned}
$$

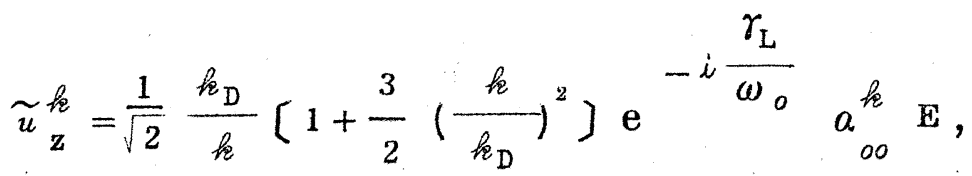

$$
\begin{aligned}
& \widetilde{T}^{k}=\frac{2}{3}\left[1+3\left(\frac{k}{k_{D}}\right)^{2}\right] \mathrm{e}^{-i \frac{\gamma_{L}}{\omega_{0}}}\left(\frac{h_{\mathrm{D}}}{k}\right)^{2} a_{o 0} \mathrm{E} \text {, } \\
& \widetilde{\mathrm{S}}_{\mathrm{ZZ}}^{\ell}=\frac{4}{3}\left[1+3\left(\frac{k}{\ell_{\mathrm{D}}}\right)^{2}\right] \mathrm{e}^{-i \frac{\gamma_{\mathrm{L}}}{\omega_{0}}\left(\frac{k \mathrm{D}}{\ell}\right)^{2}} a_{o 0}^{k} \mathrm{E}, \\
& \tilde{q}_{\mathrm{z}}^{k}=\frac{3}{\sqrt{2}} \frac{k}{h_{\mathrm{D}}} \mathrm{e} \mathrm{i}^{i \frac{\gamma_{\mathrm{L}}}{\omega_{0}}\left(\frac{k_{\mathrm{D}}}{h}\right)^{2}} a_{o 0}^{k} \mathrm{E} \text {, }
\end{aligned}
$$

を得る。各mode は Landau damping のためK number density fluctuation と少し phaseがずれるととが判る。 $a_{\mathrm{r}_{e}}$ の real part が各 moment wave と number density fluctuation の振巾の比を与える。 $\widetilde{u}_{\mathrm{z}}^{\ell}$ のk $\rightarrow 0$ での発散は次のよ

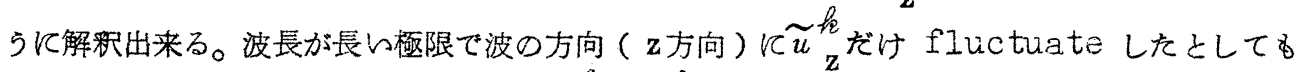
density は無限小しか変化しない。学 は差し引かれている。

上で得られた expressionは $\$ 6 て ゙$ 導かれた integrated moment eg.を満たして いる。

Collision 考慮する場合は, collision processによる phase shiftが在る 計算が複雑で，特に興昧有る結果も得られないので省略する。

\section{$\$ 8$ Effect of Ion - Electron Collision}

今迄イオンは唯 charge neutrality 保つ作用しか持たなった。しかし collision process の plasma frequency K及㴗す影響を考える時は, 電子一電子衝突のみでな

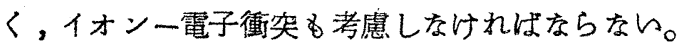


Moment Method for Wave Phenomena in a Plasma

$$
\frac{4 \pi n_{0} \mathrm{e}^{2}}{m} \gg \frac{4 \pi n_{o} \mathrm{e}^{2}}{M},
$$

M: ion mass.

従ってイオンの運動は考えなくても良い。すをねち

$$
\frac{m}{M}=0
$$

と採れる。てのために collision operator $(3,7)$ は

$$
J_{e-i} \phi\left(\vec{v}_{1}\right)=\int\left[\phi\left(\vec{v}_{1}\right)-\phi\left(\vec{v}_{1}^{\prime}\right)\right] v_{1} I\left(v_{1}, \chi\right) d \Omega(8,3)
$$

K reduce する。 collision term $(3,8)$ 㤌

$$
\left(\frac{\partial \phi}{\partial t}\right)_{c o \ell \ell}=-\sum_{n, m, \ell}\left(\lambda_{n_{m}}^{(\ell)}+\Lambda_{n_{m}}^{(\ell)}\right) a_{n_{e}} \Psi_{m \ell} \cdot(8.4)
$$

rこK

$$
\begin{aligned}
& \lambda_{n_{m}}^{(\ell)}=n_{o}\left(\Psi_{n \ell}, \mathrm{J} \Psi_{m e}\right), \\
& \Lambda_{n_{m}}^{(\ell)}=n_{o}\left(\Psi_{n_{e}}, \mathrm{~J}{ }_{e-i} \Psi_{m \ell}\right) .
\end{aligned}
$$

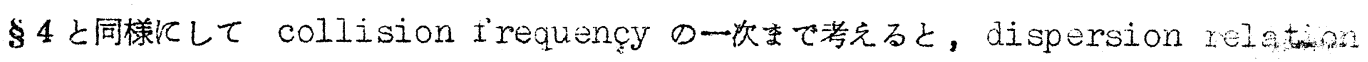
relation 4

$$
1=\alpha_{o o}+\sum_{\mathbf{r}_{e}} \overbrace{\beta_{o o v} \mathbf{v}_{e}} \alpha_{\mathbf{r}_{e}}
$$

となる。とてに

$$
\begin{aligned}
& \widetilde{\beta_{\text {OO } \mathrm{V} e}}=+i \\
& \times\left(\frac{\omega}{\omega_{0}}\right)^{2}+\Delta 5
\end{aligned}
$$

$$
\left(\frac{\omega}{\omega_{0}}\right)^{2}=-2 \lambda^{2} \mathrm{I}_{00}+i \frac{2 \lambda^{4}}{\omega} \sum_{\mathrm{rs} e}\left(\lambda_{\mathrm{rs}}^{(\ell)}+\Lambda_{\mathrm{rs}}^{\ell)}\right) \mathrm{J}_{\mathrm{s} e} \mathrm{~J}_{\mathrm{r} e}
$$


$\Sigma^{\prime}$ は $\mathrm{s}=\mathrm{r}=\ell=0$ を除くことを意味する。 $\Lambda_{\mathrm{rs}}^{(\ell)}$ の計算壮 Appendix G゙与えられる。 結果は

$$
\Lambda_{\mathrm{rs}}^{(e)} \equiv \tilde{\Lambda}_{\mathrm{rs}}^{(\ell)} / \mathrm{N}_{\mathrm{r} e} \mathrm{~N}_{\mathrm{rs}}
$$

と書く時

$$
\left.\begin{array}{c}
\tilde{\Lambda}_{\mathrm{rs}}^{(0)}=0 \quad(\ell=0) \\
\sum s_{t} \tilde{\Lambda}_{\mathrm{rs}}^{(\ell)}=\sqrt{2} \overline{\mathrm{K}}^{\prime} \frac{\Gamma(\ell+2)}{2 \ell+1} \frac{(1-\Delta)^{-\frac{3}{2}}(1-t)^{-\frac{3}{2}}}{(1-\Delta t) \ell} n_{0},(\ell \neq 0)
\end{array}\right\}
$$

rこK

$$
\overline{\mathrm{K}}^{\prime}=\left(\frac{\mathrm{e}_{1} \mathrm{e}_{2}}{\kappa \mathrm{T}_{0}}\right)^{2}\left(\frac{\pi \kappa \mathrm{T}_{0}}{m}\right)^{1 / 2} \ln \left(\frac{\kappa \mathrm{T} \ell_{\mathrm{D}}}{\mathrm{e}_{1} \mathrm{e}_{2}}\right) .
$$

電子一電子 collision invariance（4.18）に対応して我々の場合は

$$
A_{\mathrm{rs}}^{(0)}=0
$$

(8.8)を evaluateする時, 前の (4.21)に対応して
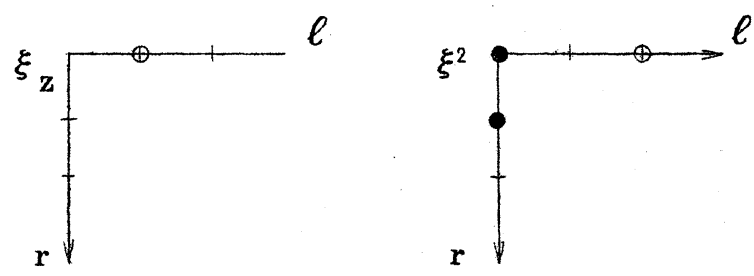

となるととに注意すると

$$
\mathrm{J}_{\mathbf{r} e}=\frac{i}{\sqrt{2} \lambda^{2}} \delta_{o \mathbf{r}} \delta_{1 \ell}-\frac{1}{\sqrt{3} \lambda^{3}} \delta_{o \mathbf{r}} \delta_{2 \ell}+\mathbf{0}\left(\frac{1}{\lambda^{4}}\right) .
$$

従って

$$
\sum_{\mathrm{rs} e}^{\prime}\left(\lambda_{\mathrm{rs}}^{(\ell)}+\Lambda_{\mathrm{rs}}^{(\ell)}\right) \mathrm{J}_{\mathrm{r}_{e}} \mathrm{~J}_{\mathrm{s}_{e}}=-\frac{\Lambda_{o o}^{(1)}}{2 \lambda^{4}}+\frac{\left(\lambda_{o o}^{(2)}+\Lambda_{o o}^{(2)}\right)}{3 \lambda^{6}}+0\left(\frac{1}{\lambda^{8}}\right)
$$


これを( 8.8$)$ に代入して

$$
\left(\frac{\omega}{\omega_{0}}\right)^{2}=1-\frac{3}{2 \lambda^{2}}-i \frac{1}{\omega}\left[\Lambda_{o 0}^{(1)}-\frac{2}{3 \lambda^{2}}\left(\lambda_{00}^{(2)}+\Lambda_{o 0}^{(2)}\right)\right]
$$

従って

$$
\begin{array}{r}
\left(\frac{\omega}{\omega_{0}}\right)^{2}=1+3\left(\frac{k}{\ell_{D}}\right)^{2}-\frac{i \Lambda_{o O}^{(1)}}{\omega_{0}}-\frac{4}{3}\left(\frac{k}{k_{D}}\right)^{2}\left(\frac{i \lambda_{00}^{(2)}}{\omega_{0}}+\frac{i \Lambda_{o 0}^{(2)}}{\omega_{o}}\right) . \\
(8.14)
\end{array}
$$

てれから

$$
\begin{aligned}
& \left(\frac{\omega_{\mathrm{r}}}{\omega_{o}}\right)^{2}=1+3\left(\frac{k}{k_{\mathrm{D}}}\right)^{2}, \\
& \omega_{i}=\frac{1}{2} \Lambda_{o 0}^{(1)}+\frac{2}{3}\left(\frac{k}{k_{\mathrm{D}}}\right)^{2}\left(\lambda_{o O}^{(2)}+\Lambda_{o 0}^{(2)}\right)
\end{aligned}
$$

(8、10)を用いて

$$
\Lambda_{o o}^{(1)}=\frac{4 \sqrt{2}}{3} n_{o} \overline{\mathrm{K}}^{\prime}, \Lambda_{o o}^{(2)}=\frac{8 \sqrt{2}}{5} n_{o} \overline{\mathrm{K}}^{\prime} .
$$

又Iの Tableから

$$
\lambda_{o o}^{(2)}=\frac{8}{5} n_{o} \overline{\mathrm{K}}
$$

$$
\mathrm{e}_{1}=\mathrm{e}_{2} \text { とすると } \overline{\mathrm{K}}=\overline{\mathrm{K}}^{\prime} \text { 。 }
$$

(8.16),(8.17)を(8.15)に代入すると

$$
\dot{\omega}_{i}=\frac{2 \sqrt{2}}{3} n_{0} \overline{\mathrm{K}}+\frac{16}{15}(1+\sqrt{2}) n_{0} \overline{\mathrm{K}}\left(\frac{k}{\ell_{\mathrm{D}}}\right)^{2} .
$$

（8.15）に於ける $\frac{1}{2} \Lambda_{\text {oo }}^{(1)}$ はイオン一電子衝乫による momentum変化のためために生 じ項である。従って電子一電子衝突の際には現われなかった項である。又虾の order の 項はイオンの影響のために約 2 倍になる。

§5.と同じ議論によると，波は進行方向の momentumをイオンとのcollision で失んな 
がら dampすると䦙時に, 進行方向とそれに垂直方向との温度差を消すような抎散をしながら 振動しているととが判る。

終りに，で指導下さった木原先生, 有益な討論をして下さった水野氏はじめ木原研究窒の皆さ んに梁く感謝したします。

$$
\text { Appendix A }
$$

(a) $\quad \alpha_{\mathrm{r}_{e}}$ とJ${ }_{\mathrm{r}_{e}}$ との関係

定義：

$$
\begin{aligned}
& \alpha_{\mathrm{r}_{e}}=-\left(\frac{\omega_{o}}{\omega}\right)^{2} 2 \lambda^{2}\left(\frac{i \xi_{\mathrm{z}}}{\lambda-i \xi_{\mathrm{z}}}, \Psi_{\mathbf{r}_{e}}\right) \\
&\left.=\left(\frac{\ell_{\mathrm{D}}}{\ell}\right)^{2}\left(\frac{i \xi_{\mathrm{z}}}{\lambda-i \xi_{\mathrm{z}}}\right), \Psi_{\mathbf{r}_{e}}\right), \\
& \mathrm{J}_{\mathbf{r}_{e}}=\left(\frac{1}{\lambda-i \xi_{\mathbf{z}}}, \Psi_{\mathbf{r}_{e}}\right) .
\end{aligned}
$$

$\alpha \mathbf{r} e^{\text {を変形すると }}$

$$
\begin{aligned}
\alpha_{\mathrm{r}_{e}} & =-\left(\frac{\omega_{0}}{\omega}\right)^{2} 2 \lambda^{2}\left(\lambda \mathrm{J}_{\mathrm{r}_{e}}-\delta_{\mathrm{r} o} \delta_{e o}\right) \\
& =\left(\frac{k_{\mathrm{D}}}{h}\right)^{2}\left(\lambda \mathrm{J}_{\mathrm{r} e}-\delta_{o \mathrm{r}} \delta_{o \ell}\right) .
\end{aligned}
$$

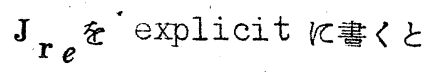

$$
\begin{aligned}
& \mathrm{J}_{\mathbf{r}_{e}}=\frac{1}{n_{o}} \int f(0) \frac{\Psi_{\mathrm{r}_{e}}}{\lambda-i \xi_{\mathrm{z}}} d \overrightarrow{\mathrm{v}} \equiv \frac{1}{\mathrm{~N}_{\mathrm{r}_{e}}} \widetilde{\mathrm{J}}_{\mathrm{r}_{e}} \\
& \widetilde{\mathrm{J}}_{\mathbf{r}_{e}}=\frac{1}{\pi^{\frac{3}{2}}} \int \mathrm{e}^{-\xi^{2}} \frac{\varphi_{\mathbf{r}_{e}}}{\lambda-i \xi_{\mathrm{z}}} d \xi=\left(\frac{1}{\lambda-i \xi_{\mathrm{z}}}, \varphi_{\mathrm{r}_{e}}\right)
\end{aligned}
$$




\section{Moment Wethod for Wave Phenomena in a Plasma}

特 $R r=\ell=0$ の時, $\mathrm{N}_{o 0}=1$ 加

$$
\widetilde{J}_{00}=\mathrm{J}_{00} .
$$

(A・3)の積分で principal part と poleての寄与を考充て $(\lambda \rightarrow \infty)$ h $\rightarrow 0$ の漸近 形を求めると

$$
\mathrm{J}_{o o}(\lambda)=\frac{1}{\lambda}\left[1-\frac{1}{2 \lambda^{2}}+\frac{3}{4 \lambda^{4}}-\frac{15}{8 \lambda^{6}}+\cdots\right]-\sqrt{\pi} \mathrm{e}^{\lambda^{2}} .
$$

(b) $\widetilde{\mathrm{J}}_{\mathbf{r}}$ の母函数 $^{-}$

Sonine の多項式の母函数：

$$
\begin{aligned}
& (1-s)^{-\left(\ell+\frac{3}{2}\right)} \quad \exp \left(-\mathrm{S} \xi^{2}\right)=\sum_{\mathbf{r}=0}^{\infty} s^{\mathbf{r}} \mathrm{S}^{(\mathbf{r})}\left(\xi^{2}\right), \\
& \mathrm{S}=\frac{s}{1-s} .
\end{aligned}
$$

を用牝 $\widetilde{\mathrm{J}} \mathrm{r} e$ 觉対与る母圂数を求好。

$$
\begin{aligned}
& (\mathrm{b} \cdot 1) \quad \ell=0 \\
& \mathrm{P}_{0}=1 \text { なので }(2 \cdot 3) \text { から } \\
& \varphi_{\mathrm{r} O}=\mathrm{S}_{\mathrm{I} / \mathrm{r}}^{(\mathbf{r})}\left(\boldsymbol{\xi}^{2}\right) .
\end{aligned}
$$

従って $(\mathrm{A} \cdot 5)$ Kょり

$$
\sum_{\mathbf{r}=0}^{\infty} s^{\mathbf{r}} \varphi_{\mathrm{ro}_{0}}=(1-s)^{-\frac{3}{2}} \mathrm{e}^{-s \xi^{2}} .
$$

これと $(\mathbf{A} \cdot 3)$ とから

$$
\begin{aligned}
& \sum_{\mathrm{r}}{ }_{s}^{\mathrm{r}} \tilde{\mathrm{J}}_{\mathrm{r} o}=(1-s)^{-\frac{3}{2}}\left(\frac{1}{\lambda-i \xi_{\mathrm{z}}}, \mathrm{e}^{-\mathrm{s} \xi^{2}}\right) . \\
& \left(\frac{1}{\lambda-i \xi_{\mathrm{z}}}, \mathrm{e}^{-\mathrm{s} \xi^{2}}\right)=\frac{1}{\pi^{\frac{3}{2}}} \int \frac{\mathrm{e}^{-(1+s) \xi^{2}}}{\lambda-i \xi_{\mathrm{z}}} d \vec{\xi}=\frac{1}{1+\mathrm{s}} \mathrm{J}_{o o}\left(\lambda^{\prime}\right) \\
& \lambda^{\prime}=\sqrt{1+s} \lambda .
\end{aligned}
$$


従って

$$
\sum_{r=0}^{\infty} s^{\mathbf{r}} \widetilde{J}_{\mathbf{r} o}=(1-s)^{-\frac{1}{2}} J_{o o}\left((1-s)^{-\frac{1}{2}} \lambda\right) . \quad(A \cdot 6)
$$

(b.2) $\quad \ell=1$

上と同様にして

$$
\begin{aligned}
& \sum_{\mathbf{r}} s^{\mathbf{r}} \varphi_{\mathrm{r} 1}=(1-\Delta)^{-\frac{5}{2}} \mathrm{e}^{-s \xi^{2}} \xi_{\mathrm{z}} \\
& \left(\frac{i \xi_{\mathrm{z}}}{\lambda-i \xi_{\mathrm{z}}}, \mathrm{e}^{-s \xi^{2}}\right)=\lambda\left(\frac{1}{\lambda-i \xi_{\mathrm{z}}}, \mathrm{e}^{-s \xi^{2}}\right)-\left(1, \mathrm{e}^{-s \xi^{2}}\right) \\
& \left(1, \mathrm{e}^{-s \xi^{2}}\right)=(1+s)^{-\frac{3}{2}}=(1-\Delta)^{\frac{3}{2}}
\end{aligned}
$$

従って

$$
\sum_{\mathbf{r}=0}^{\infty} s^{\mathbf{r}} \widetilde{J}_{\mathbf{r} I}=\frac{i}{1-s}-i(1-s)^{-\frac{3}{2}} \lambda J_{o o}\left((1-s)^{-\frac{1}{2}} \lambda\right) .(\mathrm{A} \cdot 7)
$$

$(\mathrm{b} \cdot 3) \quad \ell=2$

$$
\begin{aligned}
& \sum_{\mathrm{r}} s^{\mathrm{r}} \varphi_{\mathrm{r} 2}=(1-s)^{-\frac{7}{2}}\left(\frac{3}{2} \xi_{\mathrm{z}}^{2}-\frac{\xi^{2}}{2}\right) \mathrm{e}^{-\mathrm{s} \xi^{2}} . \quad \text { (A.8) } \\
& \frac{\xi_{\mathrm{z}}^{2}}{\lambda-i \xi_{\mathrm{z}}}=-\lambda^{2} \frac{1}{\lambda-i \xi_{\mathrm{z}}}+\lambda+i \xi_{\mathrm{z}} \\
& \left(\frac{\xi_{\mathrm{z}}^{2}}{\lambda-i \xi_{\mathrm{z}}}, \mathrm{e}\right)=-\lambda^{2}(1-\Delta) \mathrm{J}_{o o}\left((1-\Delta)^{-\frac{1}{2}} \lambda\right) \\
& +\lambda(1-s)^{\frac{3}{2}} .
\end{aligned}
$$


Moment Wethod for Wave Phenomena in a Plasma

$$
\begin{aligned}
\left(\frac{\xi^{2}}{\lambda-i \xi_{\mathrm{z}}}, \mathrm{e}^{-\mathrm{s} \xi^{2}}\right) & =-\frac{d}{d \mathrm{~S}}\left(\frac{1}{\lambda-i \xi_{\mathrm{z}}}, \mathrm{e}^{-\mathrm{S} \xi^{2}}\right) \\
& =-\frac{d}{d \mathrm{~S}}\left[\frac{\mathrm{J}_{o o} \sqrt{1+\mathrm{S}} \lambda}{1+\mathrm{S}}\right] .
\end{aligned}
$$

一方簡単な計算加

$$
\frac{d \mathrm{~J}_{o o}(\lambda)}{d \lambda}=2\left[\lambda \mathrm{J}_{o o}(\lambda)-1\right]
$$

が示される。これを用いると

$$
\begin{aligned}
& \left(\frac{\xi^{2}}{\lambda-i \xi_{\mathrm{z}}}, \mathrm{e}^{-\mathrm{S} \xi^{2}}\right)=\frac{1-(1+\mathrm{S})^{2} \lambda^{2}}{(1+\mathrm{S})^{2}} \mathrm{~J}_{o o}(\sqrt{1+S} \lambda)+\frac{\lambda}{(1+\mathrm{S})^{\frac{3}{2}}} \\
& \quad=\left[(1-\Delta)^{2}-\lambda(1-s)\right] \mathrm{J}_{o o}\left((1-\Delta)^{-\frac{1}{2}} \lambda\right)+(1-\Delta)^{\frac{3}{2}} \lambda(\mathrm{A} \cdot 10)
\end{aligned}
$$

$(\mathbf{A} \cdot 8) ，(\mathbf{A}, 9) ，(\mathrm{~A}, 10)$ を用的て

$$
\sum s^{\mathbf{r}} \widetilde{J}_{\mathbf{r} 2}=(1-s)^{-2} \lambda-\left[( 1 - s ^ { - \frac { 5 } { 2 } } \lambda ^ { 2 } + \frac { ( 1 - s ^ { - \frac { 3 } { 2 } } } { 2 } ] J _ { o o } \left(\left(1-\frac{1}{s^{2}} \lambda\right)\right.\right.
$$

$$
(A \cdot 11)
$$

( b.4) をと め

(A.4 )の exponential term は Landau dampingt与える。以下この項を考虑 しないでで得た結果をまとめる。それを附け加えるととは容易である。

( A. 4$)$ を用的て $(\mathbf{A} \cdot 6) ，(\mathbf{A} \cdot 7) ，(\mathbf{A} \cdot 7) ，(\mathbf{A} \cdot 11)$ を書くと

$$
\begin{aligned}
& \sum_{\mathbf{r}=0}^{\infty} \Delta^{\mathbf{r} \widetilde{J}_{\mathbf{r} o}}=\frac{1}{\lambda}\left[1-\frac{1-s}{2 \lambda^{2}}+\frac{3(1-\Delta)^{2}}{4 \lambda^{4}} \cdots\right], \quad(\mathrm{A} \cdot 12) \\
& \sum_{\mathbf{r}=0}^{\infty} s^{\mathbf{r}} \widetilde{J}_{\mathbf{r} 1}=\frac{i}{2 \lambda^{2}}\left[1-\frac{3(1-s)}{2 \lambda^{2}}+\frac{15(1-s)^{2}}{4 \lambda^{4}} \cdots\right),
\end{aligned}
$$




$$
\sum_{\mathrm{r}=0}^{\infty} \Delta \mathrm{r}_{\mathrm{J}_{\mathrm{r} 2}}=-\frac{1}{2 \lambda^{3}}\left[1-\frac{6(1-\Delta)}{2 \lambda^{2}}+\cdots\right]
$$

を得る。もっと compact好まとると

$$
\begin{aligned}
& \sum_{\mathrm{r}=0}^{\infty} s \widetilde{J}_{\mathrm{r} o}=\frac{1}{\lambda} \sum_{n=0}^{\infty}(-1)^{n} \frac{\Gamma\left(n+\frac{1}{2}\right)}{\sqrt{\pi}} \frac{(1-s)^{n}}{\lambda^{2 n}},(\mathbf{A} \cdot 15) \\
& \sum_{\mathbf{r}=0}^{\infty} s \mathbf{r} \widetilde{J}_{\mathbf{r} 1}=\frac{i}{2 \lambda^{2}} \sum_{n=0}^{\infty}(-1)^{n} \frac{2}{\sqrt{\pi}} \Gamma\left(n+\frac{3}{2}\right) \frac{(1-s)^{n}}{\lambda^{2 n}}, \text { (A. 16) } \\
& \sum_{\mathbf{r}=0}^{\infty} s \mathbf{r} \widetilde{J}_{\mathbf{r}^{2}}=-\frac{1}{2 \lambda^{3}} \sum_{n=0}^{\infty}(-1)^{n} \frac{2}{\sqrt{\pi}}(n+1) \Gamma\left(n+\frac{3}{2}\right) \frac{(1-s)^{n}}{\lambda^{2 n}} \\
& \text { (A. } 17 \text { ) }
\end{aligned}
$$

定義： $\mathrm{I}_{\mathbf{r} e}=\left(\frac{i \xi_{\mathrm{z}}}{\lambda-i \xi_{\mathrm{z}}}, \Psi_{\mathbf{r}_{e}}\right)$.

変形すると $\quad \mathrm{I}_{\mathrm{r}_{e}}=\lambda \mathrm{J}_{\mathrm{r}_{e}}-\delta_{o \mathrm{r}} \delta_{o} o_{e}$

$$
\mathrm{I}_{\mathrm{r}_{e}} \equiv \widetilde{\mathrm{I}}_{\mathrm{r}_{e}} / \mathrm{N}_{\mathrm{r}_{e}}
$$

とすると

$$
\widetilde{\mathrm{I}}_{r_{e}}=\lambda \widetilde{\mathrm{J}}_{r e}-\delta_{o r} \delta_{o_{e}} .
$$

特泟

$$
I_{o o}=\widetilde{I}_{00}=\lambda \widetilde{J}_{00}-1
$$

(A.15)を航て

$$
\mathrm{I}_{o \circ}=-\frac{1}{2 \lambda^{2}} \sum_{n=0}^{\infty}(-1)^{n} \frac{2}{\sqrt{\pi}} \Gamma\left(n+\frac{3}{2}\right) \frac{1}{\lambda^{2 n}} \cdot(\mathrm{A} \cdot 18)
$$

又は., (A.12)を用いて

$$
I_{O O}=-\frac{1}{2 \lambda^{2}}\left[1-\frac{3}{2 \lambda^{2}}+\frac{15}{4 \lambda^{4}}-\cdots \cdot \cdot\right] \cdot(\mathrm{A} \cdot 19)
$$


Moment Method for Wave Phenomena in a Plasma

Appendix B.'

Derivation of a Recurrence Formula

定義：

$$
\begin{aligned}
& \alpha^{2}=\frac{m}{2 \kappa \mathrm{T}_{0}}, \xi=\alpha v, \\
& \varphi_{\mathbf{r} \ell}=\xi^{\ell}{ }_{\mathrm{P}_{\ell}}(\mathrm{z}) \mathrm{S}_{\ell+\frac{1}{2}\left(\xi^{2}\right),}^{(\mathbf{r})} \\
& \mathrm{z}=\xi_{\mathrm{z}} / \xi .
\end{aligned}
$$

公式：

$$
\begin{aligned}
& (2 \ell+1) \mathrm{P}_{\ell}=(\ell+1) \mathrm{P}_{\ell+1}+\ell \mathrm{P}_{\ell-1} \\
& \mathrm{~s}_{\ell+\frac{1}{2}}^{(\mathrm{r})}=\mathrm{s}_{(\ell+1)+\frac{1}{2}-\mathrm{S}_{(\mathrm{r})}^{(\mathrm{r}-1)}}^{(\mathrm{B} \cdot 2)} \\
& \xi^{2} \mathrm{~S}_{m}^{(n)}=(n+m) \mathrm{s}_{m-1}^{(m)}-(n+1) \mathrm{s}_{m-1}^{(n+1)}
\end{aligned}
$$

(B.1)を用いて

$$
\xi_{\mathrm{z}} \varphi_{\mathrm{r} \ell}=\xi^{\ell+1} \mathrm{zP}_{\ell} \mathrm{s}_{\ell+\frac{1}{2}}^{(\mathrm{r})}
$$

(B.2) 学用故え

$$
\begin{gathered}
(2 \ell+1) \xi_{\mathrm{z}} \varphi_{\mathrm{r} \ell}=\xi^{\ell+1}\left[(\ell+1) \mathrm{P}_{\ell+1}+\ell \mathrm{P}_{\ell-1}\right] \mathrm{s}^{(\mathbf{r})} \\
=(\ell+1) \xi^{\ell+1} \mathrm{P}_{\ell+1} \mathrm{~s}_{\ell+\frac{1}{2}}^{(\mathrm{r})}+\ell \xi^{\ell-1} \mathrm{P}_{\ell-1} \xi^{2} \mathrm{~s}_{\ell+\frac{1}{2}}^{(\mathbf{r})}
\end{gathered}
$$

(B.3)，(B.4)を用いて (B.1)を考えると

$(2 \ell+1) \xi_{\mathrm{z}} \varphi_{\mathrm{r} \ell}=(\ell+1)\left[\varphi_{\mathrm{r}, \ell+1}-\varphi_{\mathrm{r}-1, \ell+1}\right]$

$$
+\ell\left[\left(\ell+\mathbf{r}+\frac{1}{2}\right) \varphi_{\mathbf{r}, \ell-1}-(\mathbf{r}+1) \varphi_{\mathbf{r}+1, \ell-1}\right]
$$




$$
\Psi_{\mathbf{r}_{e}}=\varphi_{\mathbf{r}_{\boldsymbol{\ell}}} / \mathrm{N}_{\mathbf{r}_{\boldsymbol{e}}} \text { を考慮して (B.5)を畫を換え, normalization }
$$

constant $\mathrm{N}_{\mathbf{r}_{e}}$ の定義 ( 2.5$)$ を用いて整理すると求める漸化式を得る。

$$
\xi_{\mathrm{z}} \Psi_{\mathbf{r}_{e}}=\mathrm{A}_{\mathrm{r}_{e}} \Psi_{\mathrm{r} \cdot \ell+1}{ }^{-\mathrm{B}_{\mathrm{r}_{e}} \Psi_{\mathrm{r}-1, \ell+1}+\mathrm{C}_{\mathbf{r}} e^{\Psi_{\mathrm{r}} \cdot \ell-1}-\mathrm{D}_{\mathbf{r}_{e}} \Psi_{\mathrm{r}+1, \ell-1}}
$$

ことに

$$
\begin{gathered}
\mathrm{A}_{\mathrm{r}_{e}}=(\ell+1) \sqrt{\frac{\ell+\mathrm{r}+\frac{3}{2}}{(2 \ell+1)(2 \ell+3)}}, \mathrm{B}_{\mathrm{r} \ell}=(\ell+1) \sqrt{\frac{\mathrm{r}}{(2 \ell+1)(2 \ell+3)}} \\
\mathrm{C}_{\mathrm{r} e}=\ell \sqrt{\frac{\ell+\mathrm{r}+\frac{1}{2}}{(2 \ell-1)(2 \ell+1)}}, \mathrm{D}_{\mathrm{r}_{e}}=\ell \sqrt{\frac{\mathrm{r}+1}{(2 \ell-1)(2 \ell+1)}}
\end{gathered}
$$

これら係数の間に

$$
\mathrm{A}_{\mathbf{r} e}=\mathrm{C}_{\mathbf{r} \cdot \ell+1}, \quad \mathrm{~B}_{\mathbf{r}+1, \ell}=\mathrm{D}_{\mathbf{r}, \ell+1}
$$

が成り立つ。

\section{Appendix C}

$$
\text { Évaluation of }\left(\varphi \mathrm{p} \ell,{ }^{\mathrm{J}} e-i \varphi g \ell\right)
$$

電子一イオン collision operatorの定義 ( 8.3 ) と Sonine の多項式の母函数 ( A

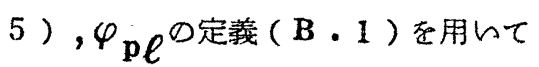

$$
\begin{aligned}
& \sum_{g=0}^{\infty} s^{q} \mathrm{~J} e_{-i} \varphi_{q \ell}=(1-s)^{-\left(\ell+\frac{3}{2}\right)} \int\left[\xi^{\ell} \ell_{\mathrm{P}} \mathrm{e}^{-\mathrm{s} \xi^{2}}-\xi^{\prime} \ell_{\mathrm{P}_{e}^{\prime}} \mathrm{e}^{-s \xi^{\prime 2}}\right] \\
& \times v I(v, \chi) d \Omega, \\
& m / \mathbf{M}=0 \text { から } \\
& \xi=\xi^{\prime} \text {. } \\
& \text { これを用いると（C・1) は }
\end{aligned}
$$


Moment Method for Wave Phenomena in a Plasma

$$
\begin{aligned}
& \sum_{q=0}^{\infty} s^{q} J_{e-i} \varphi_{g \ell}=(1-s)^{-\left(\ell+\frac{3}{2}\right)} e^{-s \xi^{2}} \xi^{\ell} A_{\ell}, \quad \text { (C.2) } \\
& { }^{\mathbf{A}_{\ell}}=\iint\left[\mathrm{P}_{\ell}(\cos \theta)-\mathrm{P}_{\ell}\left(\cos \theta^{\prime}\right)\right] v \mathrm{I}(v, \chi) \sin \chi d \chi d \varepsilon \cdot(\mathrm{C} .3) \\
& \text { こてに } \quad \cos \theta=\xi_{z /}
\end{aligned}
$$

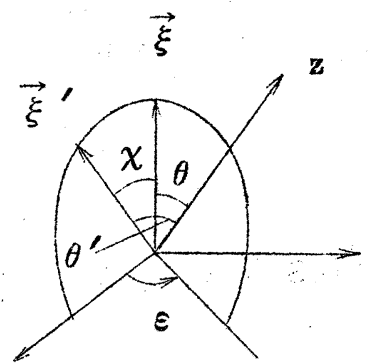

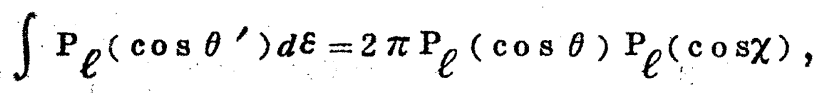

$$
\begin{aligned}
& { }^{\mathrm{A}} \ell^{=2 \pi \mathrm{P}} \ell^{\left.(\cos \theta) \int\left[1-\mathrm{P}_{\ell}(\cos \chi)\right] v \mathrm{I}(v, \chi) \sin \chi d \chi \ldots \text { (C . } 4\right)} \\
& 1-P_{\ell}(\cos \chi)=(1-\cos \chi) \frac{\ell(\ell+1)}{2} \quad(\mathrm{C} .5)
\end{aligned}
$$

cross section は

$$
v I(v, \chi)=\frac{1}{2 \sqrt{2 \pi}}\left(\frac{\mathbf{e}_{1} \mathbf{e}_{2}}{\kappa \mathrm{T}_{0}}\right)\left(\frac{\pi{ }_{k} \mathrm{~T}_{o}}{m}\right)^{\frac{1}{2}} \frac{1}{\xi^{3}(1-\cos \chi)^{2}}
$$

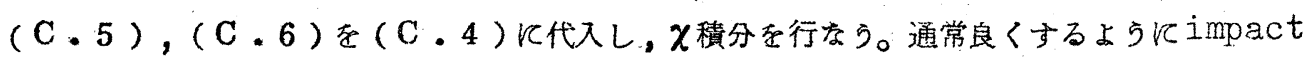
parameter が Debye length K相当する所で cutする。すると

$$
\begin{aligned}
& \mathrm{A}_{\ell}=\sqrt{2 \pi} \overline{\mathrm{K}}^{\prime} \frac{\ell(\ell+1)}{2} \frac{\mathrm{P}_{\ell}(\cos \theta)}{\xi^{3}}, \\
& \overline{\mathrm{K}}^{\prime}=\left(\frac{\mathrm{e}_{1} \mathrm{e}_{2}}{\kappa \mathrm{T}_{0}}\right)^{2}\left(\frac{\pi \kappa \mathrm{T}_{o}}{m}\right)^{\frac{1}{2}} \ln \left(\frac{\kappa \mathrm{T}_{o} \ell_{\mathrm{D}}}{\mathrm{e}_{1} \mathrm{e}_{2}}\right) . \quad(\mathrm{C} \cdot 7)
\end{aligned}
$$


これと $(\mathrm{C} \cdot 2)$ とから

$$
\begin{aligned}
& \sum_{g=0}^{\infty} \Delta \delta \mathrm{J}_{e-i} \varphi_{g}=(1-\Delta)^{-\left(\ell+\frac{3}{2}\right)} \mathrm{e}^{-\mathrm{S} \xi^{2}} \xi\left(-3 \sqrt{2 \pi} \overline{\mathrm{K}}^{\prime} \frac{\ell(\ell+1)}{2} \mathrm{P}^{(\cos \theta)}\right. \\
& (\mathrm{C} \cdot 8)
\end{aligned}
$$

内積の定義を用的て

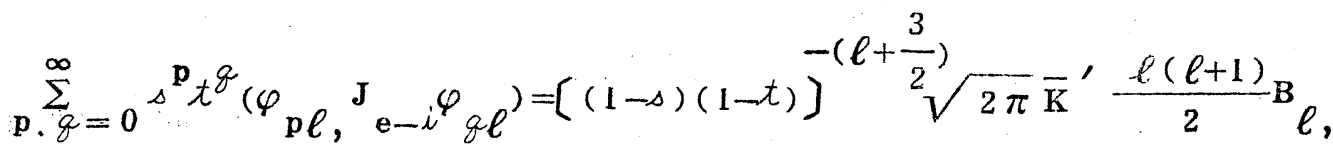

$$
\begin{aligned}
& \text { (C.9) } \\
& \mathbf{B}_{\ell}=\frac{2}{\sqrt{\pi}} \int_{-1}^{1} \mathrm{P}_{e}^{2}(\cos \theta) d(\cos \theta) \int_{0}^{\infty} \mathrm{e}^{-(1+\mathrm{S}+\mathrm{T}) \xi^{2}} \xi^{2 \ell-1} d \xi_{\bullet}(\mathrm{C} \cdot 10) \\
& \stackrel{B}{\ell}=\frac{1}{\sqrt{\pi}} \frac{2}{2 \ell+1} \frac{\Gamma(\ell)}{(I+S+T) \ell} .
\end{aligned}
$$

とれと $(\mathrm{C} \cdot .9)$ とから

$$
\begin{aligned}
& \sum_{\mathrm{p} \cdot \mathrm{g}=0}^{\infty} s^{\mathrm{p}} t^{q}\left(\varphi_{\mathrm{p} \ell},_{\mathrm{e}-i^{\varphi}}^{\mathrm{J}} q \ell^{)}\right) \sqrt{2} \overline{\mathrm{K}}^{\prime} \frac{\Gamma(\ell+2)}{2 \ell+1} \frac{[(1-\Delta)(1-t)]^{-\left(\ell+\frac{3}{2}\right)}}{(1+\mathrm{S}+\mathrm{T})^{\ell}} \\
& \mathrm{S}=\frac{s}{1-s}, \mathrm{~T}=\frac{t}{1-t} \text { を用いて }
\end{aligned}
$$

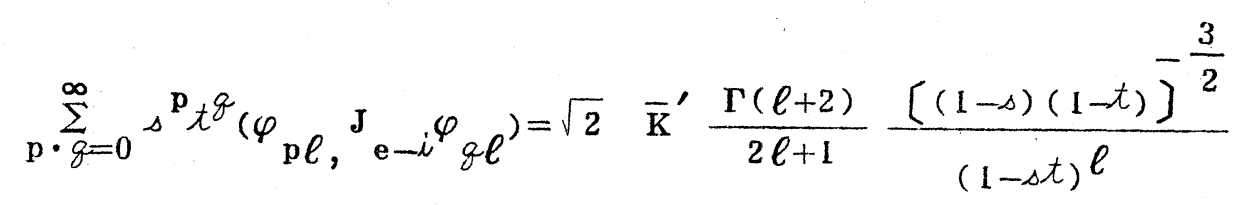

と書かれる。 
Moment Method for Wave Phenomena in a Plasma

Reference

1) M. Ogasawara : J. Phys. Soc. Japan 17 (1962) 1553, 小笠原, 本間, 木原：核融合研究 \& 8 (1962) 116, 小笠原：核融合研究 8 （1962）441.

2) C.S. Wang Chang and G. E. Uhlenbeck: Univ. Michigan Rep. (1952).

3) A.Lenard : Ann. Phys. 10. (1960) 390, R. Balescu: Phys. Fluids 3 (1960) 52, N. Rostoker and M. N. Rosenbluth: Phys. rluids 2 (1960) I.

4) Y.H. Ichikawa : Prog. Theor. Phys. 24 (1960) 1083.

5) C.R. Willis: Phys. rluids 5 (1962) 219.

6) J.E. urummond: Phys. Rev. 110 (1958) 293, 112 (1958) 1460 , L. Hower: Phys. Rev. 116 (1959) 16.

7) P.L. Bhatnager, E.P. Gross and M. Krook: Phys. Rev. 24 (1954) 511.

8) T.W.Johnston: Canad. J. Phys. 40 (1962) 1208.

9) A.Ienard and IraB. Bernstein: Phys. Rev. 112 (1958) 1456.

10) A.A.Logunov: ihur. Eksptl. i Teoret. Fiz. 20 (1950) 458.

11) J.J. and G.P. Thomson: Conduction of Electricity through Gases, Cambridge 1933, Vol. 2 p. 353.

12) V.A.Bailey: Phys. Rev. 78 (1950) 428.

13) E.P.Gross: Phys. Rev. 82 (1951) 232. 